\title{
Penicillin-binding proteins in Actinobacteria
}

\author{
Hiroshi Ogawara ${ }^{1,2}$
}

Because some Actinobacteria, especially Streptomyces species, are $\beta$-lactam-producing bacteria, they have to have some self-resistant mechanism. The $\boldsymbol{\beta}$-lactam biosynthetic gene clusters include genes for $\boldsymbol{\beta}$-lactamases and penicillin-binding proteins (PBPs), suggesting that these are involved in self-resistance. However, direct evidence for the involvement of $\boldsymbol{\beta}$-lactamases does not exist at the present time. Instead, phylogenetic analysis revealed that PBPs in Streptomyces are distinct in that Streptomyces species have much more PBPs than other Actinobacteria, and that two to three pairs of similar PBPs are present in most Streptomyces species examined. Some of these PBPs bind benzylpenicillin with very low affinity and are highly similar in their amino-acid sequences. Furthermore, other low-affinity PBPs such as SCLAV_4179 in Streptomyces clavuligerus, a $\beta$-lactam-producing Actinobacterium, may strengthen further the self-resistance against $\beta$-lactams. This review discusses the role of PBPs in resistance to benzylpenicillin in Streptomyces belonging to Actinobacteria.

The Journal of Antibiotics (2015) 68, 223-245; doi:10.1038/ja.2014.148; published online 29 October 2014

\section{INTRODUCTION}

Drug resistance is a major problem in almost all the drug-related fields. Among bacterial resistance, $\beta$-lactam-antibiotic resistance is the most prevailing and threatening area in public health, because $\beta$-lactam antibiotics have been most widely used for chemotherapy of infectious diseases even in 80 years since penicillin's discovery. ${ }^{1}$ $\beta$-Lactam antibiotic resistance is caused mainly by two mechanisms: antibiotic-degrading enzymes, $\beta$-lactamases ${ }^{2}$ and modification of target sites, penicillin-binding proteins (PBPs). ${ }^{3}$

The phylum Actinobacteria constitute one of the largest phyla within the Bacteria. ${ }^{4,5}$ Streptomyces species, which belong to Actinobacteria, are filamentous, soil-dwelling, high guanine+cytosine $(\mathrm{G}+\mathrm{C})$-content Gram-positive bacteria and are characterized by their ability to undergo complex cellular differentiation like filamentous fungi. ${ }^{6}$ In addition, Streptomyces species produce a wide variety of secondary metabolites including $\beta$-lactam antibiotics. ${ }^{7-9}$ However, Streptomyces species are prokaryotic microorganisms and unlike penicillin- and cephalosporin-producing fungi they must protect themselves from the attack of antibiotics, thus they have to have self-resistant mechanisms. ${ }^{10-13}$ In addition, Streptomyces species are known to be highly resistant to benzylpenicillin, although they are Gram-positive bacteria. This review discusses the role of PBPs in resistance to benzylpenicillin in Streptomyces belonging to Actinobacteria.

\section{PBPS}

The bacterial cell wall peptidoglycan is a three-dimensional, net-like mesh called sacculus in which glycan strands are cross-linked by peptide chains. It maintains cell shape and provides mechanical strength to resist osmotic pressure. ${ }^{14,15}$ The peptidoglycan biosynthesis is catalyzed by glycosyltransferases to polymerize the glycan chains and by transpeptidases to catalyze peptide crosslinking between two adjacent glycan chains. The transpeptidases, also called PBPs, were initially identified as their ability to bind penicillins. ${ }^{16,17}$ Depending on the structure and the catalytic activity of their N-terminal domain, they are classified into class A, B and C PBPs. ${ }^{14,18-20}$ The C-terminal domains of both class A and class B PBPs have the transpeptidase activity. In class A PBPs, the N-terminal domain is responsible for their glycosyltransferase activity, whereas in class B PBPs, the glycosyltransferase domain is lacking. Class C PBPs are also called low-MW PBPs, having the carboxypeptidase activity, and are responsible for the maturation and recycling of the peptidoglycan. ${ }^{19}$ They are not essential and are excluded from further study.

Table 1 summarizes the genome sizes, G+C contents, numbers of PBPs, types of PBPs, class A and class B PBPs and some characters of 113 Actinobacterial species including 30 Streptomyces species. Most species have both class A and class B PBPs. However, the phylogenetic distribution of PBPs among taxa is uneven; Actinomyces odontolyticus ATCC 17982 encodes two PBPs per genome, whereas S. avermitilis MA-4680 and $S$. coelicolor A3(2) have 13 PBPs. In general, Streptomyces species possess $>10$ PBPs including class A and class B PBPs. ${ }^{21}$

\section{CLASS B PBPS}

A phylogenetic tree constructed on the basis of their amino-acid sequences of 446 class B PBPs from 113 Actinobacterial species is classified into 10 clusters and is shown in Figure 1. In general, the PBPs from taxonomically related species go into the same clusters. PBPs of suborder Propionibacterineae belong to subcluster I. PBPs of the members of order Actinomycetales, such as Thermobispora, Streptosporangium, Actinomyces and Mobiluncus, form cluster II. PBPs of other members of Actinomycetales, such as Kytococcus, Kineococcus, Isoptericola, Cellulomonas, Sanguibacter, Beutenbergia, Janibacter,

${ }^{1} \mathrm{HO}$ Bio Institute, Tokyo, Japan and ${ }^{2}$ Meiji Pharmaceutical University, Tokyo, Japan

Correspondence: Professor H Ogawara, HO Bio Institute, 33-9, Yushima-2, Bunkyo-ku, Tokyo 113-0034, Japan.

E-mail: hogawara@sc5.so-net.ne.jp

Received 22 July 2014; revised 24 September 2014; accepted 30 September 2014; published online 29 October 2014 
Table 1 The numbers and types of putative PBP genes

\begin{tabular}{|c|c|c|c|c|c|c|c|c|}
\hline Bacteria & Prefix & $\begin{array}{c}\text { Genome } \\
\text { size } \\
(\mathrm{Mb})\end{array}$ & $\begin{array}{c}G+C \\
\%\end{array}$ & $\begin{array}{l}\text { No. of } \\
\text { PBP }\end{array}$ & $\begin{array}{l}\text { Type of } \\
\text { PBP }\end{array}$ & Class A PBP & Class B PBP & Comment \\
\hline $\begin{array}{l}\text { Acidimicrobium } \\
\text { ferrooxidans DSM } \\
10331\end{array}$ & AFER & 2.16 & 68.3 & 3 & B3 & & $\begin{array}{l}\text { 0089, } 0769 \\
1250\end{array}$ & $\begin{array}{l}\text { Ferrous-iron-oxidizing, moderately ther- } \\
\text { mophilic, acidophilic bacteria, isolated } \\
\text { from hot spring in Iceland, thermophile, } \\
\text { nonsporulating }\end{array}$ \\
\hline $\begin{array}{l}\text { Acidothermus cell- } \\
\text { ulolyticus } 11 \mathrm{~B}\end{array}$ & ACEL & 2.44 & 66.9 & 5 & A2; B3 & 2004,2135 & $\begin{array}{l}\text { 0020, } 0751 \\
1004\end{array}$ & $\begin{array}{l}\text { A moderately thermophilic, aerobic, cel- } \\
\text { lulolytic bacterium originally recovered } \\
\text { from the acidic hot springs }\end{array}$ \\
\hline $\begin{array}{l}\text { A. odontolyticus } \\
\text { ATCC } 17982\end{array}$ & ACTODO & 2.39 & 65.4 & 2 & B2 & & 00847,01564 & $\begin{array}{l}\text { Isolated from human dental cavity, non- } \\
\text { motile, nonsporulating, mesophile, septi- } \\
\text { cemia, oral abscesses, lung infection, eye } \\
\text { infection }\end{array}$ \\
\hline $\begin{array}{l}\text { A. urogenitalis } \\
\text { DORA_12 }\end{array}$ & Q605 & 2.6 & 68.4 & 5 & A3, B2 & $\begin{array}{l}\text { AUC00015G0001, } \\
\text { AUC00927G0001, } \\
\text { AUC01040G0002 }\end{array}$ & $\begin{array}{l}\text { AUC00266G0006 } \\
\text { AUC00451G0003 }\end{array}$ & Isolated from human urogenital tract \\
\hline $\begin{array}{l}\text { Actinoplanes mis- } \\
\text { souriensis } 431\end{array}$ & AMIS & 8.77 & 70.8 & 8 & A5; B3 & $\begin{array}{l}10850,39800 \\
60090,78300 \\
80960\end{array}$ & $\begin{array}{c}00450,15180 \\
71610\end{array}$ & $\begin{array}{l}\text { Aerobic, motile, filamentous, sporulating } \\
\text { bacterium; produces actaplanin, azacyti- } \\
\text { dine, D-xylose isomerase; degrades flavo- } \\
\text { noids and natural rubber; }\end{array}$ \\
\hline $\begin{array}{l}\text { Actinosynnema } \\
\text { mirum DSM } \\
43827\end{array}$ & AMIR & 8.25 & 73.7 & 6 & A3; B3 & $0235,5121,7034$ & $\begin{array}{c}0023,5772 \\
5886\end{array}$ & $\begin{array}{l}\text { A producer of nocardicin; Pseudonocar- } \\
\text { dineae, isolated from blade of grass from } \\
\text { Raritan River, aerobic, nonmotile, sporu- } \\
\text { lating, mesophile, chemoorganotroph }\end{array}$ \\
\hline $\begin{array}{l}\text { Amycolicicoccus } \\
\text { subflavus DQS3- } \\
9 \mathrm{~A} 1\end{array}$ & AS9A & 4.74 & 62.2 & 5 & A2; B3 & 0253,2577 & $\begin{array}{l}0033,4146 \\
4510\end{array}$ & $\begin{array}{l}\text { Isolated from a saline soil contaminated } \\
\text { with crude oil; no mycolic acids and MK-8 } \\
\text { was the major menaquinone }\end{array}$ \\
\hline $\begin{array}{l}\text { Arthrobacter aur- } \\
\text { escens TC1 }\end{array}$ & AAUR & 4.60 & 62.3 & 6 & A2; B4 & 3369,3416 & $\begin{array}{l}0030,1704 \\
3184,4181\end{array}$ & $\begin{array}{l}\text { Reduce hexavalent chromium; found in } \\
\text { extreme environments such as deep sub- } \\
\text { surface soils, arctic sea and radioactive } \\
\text { waste tanks; catabolize a variety of xeno- } \\
\text { biotic compounds; used in the } \\
\text { bioremediation }\end{array}$ \\
\hline $\begin{array}{l}\text { Atopobium parvu- } \\
\text { Ium DSM } 20469\end{array}$ & Apar & 1.54 & 45.7 & 4 & $\mathrm{~A} 1 ; \mathrm{B} 3$ & 1010 & $\begin{array}{c}0480,0673 \\
1344\end{array}$ & $\begin{array}{l}\text { Isolated from gingival crevices of the } \\
\text { human oral cavity, obligate anaerobic, } \\
\text { coccus-shaped, nonmotile, nonsporulat- } \\
\text { ing, free living, mesophile, } 37-45^{\circ} \mathrm{C}\end{array}$ \\
\hline $\begin{array}{l}\text { Beutenbergia } \\
\text { cavernae DSM } \\
12333\end{array}$ & BCAV & 4.67 & 73.1 & 5 & A2; B3 & 0604,4182 & $\begin{array}{l}0028,0389 \\
2416\end{array}$ & $\begin{array}{l}\text { Isolated from cave soil; no mycolic acid; } \\
\text { not acid-fast, nonmotile, non-spore- } \\
\text { forming and a rod-coccus growth cycle; } \\
\text { peptidoglycan contains lysine in position } \\
3 \text { of the peptide subunit and an inter- } \\
\text { peptide bridge of L-LYS+ L-Glu. }\end{array}$ \\
\hline $\begin{array}{l}\text { Bifidobacterium } \\
\text { adolescentis ATCC } \\
15703\end{array}$ & $B A D$ & 2.09 & 59.2 & 4 & $\mathrm{~A} 2 ; \mathrm{B} 2$ & 0157,1336 & 0040,1107 & $\begin{array}{l}\text { Normal inhabitants of healthy human and } \\
\text { animal intestinal tracts, anaerobic, rod- } \\
\text { shaped, nonsporulating, nonmotile, } \\
\text { intestinal microflora }\end{array}$ \\
\hline $\begin{array}{l}\text { Bifidobacterium } \\
\text { animalis subsp. } \\
\text { lactis AD011 }\end{array}$ & BLA & 1.93 & 60.5 & 3 & $\mathrm{~A} 1 ; \mathrm{B} 2$ & 0208 & 0077,0782 & $\begin{array}{l}\text { An anaerobic Gram-positive lactic acid } \\
\text { bacterium commonly found in the guts of } \\
\text { healthy humans; utilize nondigestible oli- } \\
\text { gosaccharides, nonsporulating }\end{array}$ \\
\hline $\begin{array}{l}\text { Bifidobacterium } \\
\text { animalis subsp. } \\
\text { lactis BLC1 }\end{array}$ & BLC1 & 1.94 & 60.5 & 4 & A2; B2 & 0209, 1314 & 0079,1166 & \\
\hline $\begin{array}{l}\text { Bifidobacterium } \\
\text { bifidum PRL2010 }\end{array}$ & BBPR & 2.21 & 62.7 & 4 & $\mathrm{~A} 2 ; \mathrm{B} 2$ & 0401,1743 & 0104,0550 & $\begin{array}{l}\text { Anaerobic, rod-shaped, nonmotile, } \\
\text { nonsporulating, mesophile }\end{array}$ \\
\hline \multirow{2}{*}{$\begin{array}{l}\text { Bifidobacterium } \\
\text { bifidum } \mathrm{S} 17\end{array}$} & BBIF & 2.19 & 62.8 & 4 & $A 2 ; B 2$ & 0427,1684 & 0137,0574 & \\
\hline & & 2.33 & 58.7 & 4 & A2; B2 & 0187,1410 & 0067,0606 & \\
\hline
\end{tabular}




\begin{tabular}{|c|c|c|c|c|c|c|c|c|}
\hline Bacteria & Prefix & $\begin{array}{c}\text { Genome } \\
\text { size } \\
(\mathrm{Mb})\end{array}$ & $\begin{array}{c}G+C \\
\%\end{array}$ & $\begin{array}{l}\text { No. of } \\
\text { PBP }\end{array}$ & $\begin{array}{l}\text { Type of } \\
\text { PBP }\end{array}$ & Class A PBP & Class B PBP & Comment \\
\hline $\begin{array}{l}\text { Bifidobacterium } \\
\text { breve ACS-071-V- } \\
\text { Sch8b }\end{array}$ & $\begin{array}{l}\text { HMPRE- } \\
\text { F9228 }\end{array}$ & & & & & & & $\begin{array}{l}\text { Anaerobic, rod-haped, nonmotile, non- } \\
\text { sporulating, mesophile, human vaginal } \\
\text { microflora }\end{array}$ \\
\hline $\begin{array}{l}\text { Bifidobacterium } \\
\text { breve UCC2003 }\end{array}$ & BBR & 2.42 & 58.7 & 4 & A2; B2 & 0176,0487 & 0069,1263 & $\begin{array}{l}\text { Isolated from infant nursing stool, anae- } \\
\text { robic,rod-shaped, nonsporulating, non- } \\
\text { motile, intestinal microflora, probiotic, } \\
\text { nonpathogenic }\end{array}$ \\
\hline $\begin{array}{l}\text { Bifidobacterium } \\
\text { dentium } \mathrm{Bd} 1\end{array}$ & BDP & 2.64 & 58.5 & 4 & A2; B2 & 0244,1801 & 0040,1548 & $\begin{array}{l}\text { Isolated from dental caries, anaerobic, } \\
\text { nonmotile, nonsporulating, mesophilic, } \\
\text { probiotic, nonpathogenic }\end{array}$ \\
\hline $\begin{array}{l}\text { Bifidobacterium } \\
\text { Iongum subsp. } \\
\text { infantis 157F }\end{array}$ & BLIF & 2.40 & 60.1 & 4 & $\mathrm{~A} 2 ; \mathrm{B} 2$ & 0139, 0411 & 0048,1329 & \\
\hline $\begin{array}{l}\text { Bifidobacterium } \\
\text { longum subsp. } \\
\text { infantis ATCC } \\
15697\end{array}$ & BLIJ & 2.83 & 59.9 & 4 & A2; B2 & 0174,2144 & 0078, 0867 & $\begin{array}{l}\text { Uses milk oligosaccharides, produces } \\
\text { fucosidases; anaerobic, non-halophilic, } \\
\text { Gram-positive bacterium; hexose meta- } \\
\text { bolism: phosphoketolase pathway (bifid } \\
\text { shunt), probiotic, nonpathogenic }\end{array}$ \\
\hline $\begin{array}{l}\text { Bifidobacterium } \\
\text { longum subsp. } \\
\text { longum F8 }\end{array}$ & $\mathrm{BIL}$ & 2.38 & 59.9 & 2 & $\mathrm{~A} 1 ; \mathrm{B} 1$ & 14530 & 18970 & $\begin{array}{l}\text { Anaerobic, nonsporulating, mesophilic, } \\
\text { human fecal, gastrointestinal tract, } 37^{\circ} \mathrm{C}\end{array}$ \\
\hline $\begin{array}{l}\text { Bifidobacterium } \\
\text { Iongum subsp. } \\
\text { Iongum JCM } 1217\end{array}$ & BLLJ & 2.39 & 60.3 & 4 & A2; B2 & 0150, 0394 & 0060,1278 & $\begin{array}{l}\text { Anaerobic, rod-shaped, nonsporulating, } \\
\text { mesophile, commensal }\end{array}$ \\
\hline $\begin{array}{l}\text { Brevibacterium } \\
\text { casei S18 }\end{array}$ & C272 & 3.66 & 68.1 & 4 & A3; B1 & $\begin{array}{c}\text { 05329, } 06024 \\
15065\end{array}$ & 05334 & $\begin{array}{l}\text { Isolated from human skin, aerobe, meso- } \\
\text { phile, symbiotic, commensal }\end{array}$ \\
\hline $\begin{array}{l}\text { Catenulispora } \\
\text { acidiphila DSM } \\
44928\end{array}$ & $\mathrm{CACl}$ & 10.47 & 69.8 & 8 & $\mathrm{~A} 1 ; \mathrm{B} 7$ & 1390 & $\begin{array}{l}0037,1307 \\
1448,1656 \\
5826,6659 \\
7282\end{array}$ & $\begin{array}{l}\text { Aerobic, free-living, nonmotile, acidophi- } \\
\text { lic Gram-positive bacterium; resistant to } \\
\text { lysozyme; peptidoglycan type is } \\
\text { A3gamma II-Dpm-Gly, filament shaped, } \\
\text { sporulating }\end{array}$ \\
\hline $\begin{array}{l}\text { Cellulomonas flavi- } \\
\text { gena DSM } 20109\end{array}$ & Cfla & 4.12 & 74.3 & 3 & B3 & & $\begin{array}{l}0027,1590 \\
3460\end{array}$ & $\begin{array}{l}\text { Rod-shaped, nonsporulating, nonmotile, } \\
\text { biomass degrader, cellulose degrader, } \\
\text { nitrate reducer, xylan degrader }\end{array}$ \\
\hline $\begin{array}{l}\text { Clavibacter michi- } \\
\text { ganensis subsp. } \\
\text { michiganensis } \\
\text { NCPPB } 382\end{array}$ & CMM & 3.3 & 72.7 & 4 & A2; B2 & 0915, 0919 & 0017, 1865 & $\begin{array}{l}\text { Aerobic, rod-shaped, nonmotile, nonspor- } \\
\text { ulating, mesophile, plant pathogen, } \\
\text { isolated from Lycopersicon esculentum }\end{array}$ \\
\hline $\begin{array}{l}\text { Conexibacter woe- } \\
\text { sei DSM } 14684\end{array}$ & Cwoe & 6.36 & 72.7 & 7 & $\mathrm{~A} 1 ; \mathrm{B} 6$ & 3542 & $\begin{array}{l}0016,1104 \\
1801,2661 \\
3775,5329\end{array}$ & $\begin{array}{l}\text { Isolated from forest soil, obligate aerobic, } \\
\text { rod-shaped, motile, nonsporulating, } \\
\text { mesophile, free living }\end{array}$ \\
\hline $\begin{array}{l}\text { C. diphtheriae } \\
\text { NCTC } 13129\end{array}$ & DIP & 2.49 & 53.5 & 5 & A2; B3 & 0298, 2294 & $\begin{array}{l}0055,1497 \\
1604\end{array}$ & $\begin{array}{l}\text { Gram-positive, nonmotile rods; produces } \\
\text { the diphtheria toxin; isolated from soil, } \\
\text { plant material, waste water and dairy } \\
\text { products, diphtheria, respiratory infec- } \\
\text { tion, chemoorganotroph }\end{array}$ \\
\hline $\begin{array}{l}\text { C. glutamicum } \\
\text { ATCC } 13032\end{array}$ & NCGL & 3.31 & 53.8 & 5 & A2; B3 & 0274,2884 & $\begin{array}{l}0042,1933 \\
2084\end{array}$ & $\begin{array}{l}\text { Unusual outer membrane } \sim 8 \mathrm{~nm} \text { thick; } \\
\text { mycolic acid-arabinoglactan- } \\
\text { peptidoglycan polymer form the cell wall }\end{array}$ \\
\hline $\begin{array}{l}\text { C. pseudotubercu- } \\
\text { losis 1/06-A }\end{array}$ & CP106 & 2.28 & 52.2 & 5 & A2; B3 & 0194, 1992 & $\begin{array}{l}0032,1278 \\
1380\end{array}$ & $\begin{array}{l}\text { A facultative intracellular pathogen; } \\
\text { causes caseous lymphadenitis in animals; } \\
\text { pleomorphic forms: coccoids and fila- } \\
\text { mentous rods; nonsporulating, non-cap- } \\
\text { sulated, nonmotile bacterium; it has } \\
\text { fimbriae }\end{array}$ \\
\hline $\begin{array}{l}\text { C. pseudotubercu- } \\
\text { losis } 1002\end{array}$ & CP1002 & 2.34 & 52.2 & 5 & A2; B3 & 0200, 2034 & $\begin{array}{l}0035,1298 \\
1397\end{array}$ & \\
\hline
\end{tabular}


Table 1 (Continued)

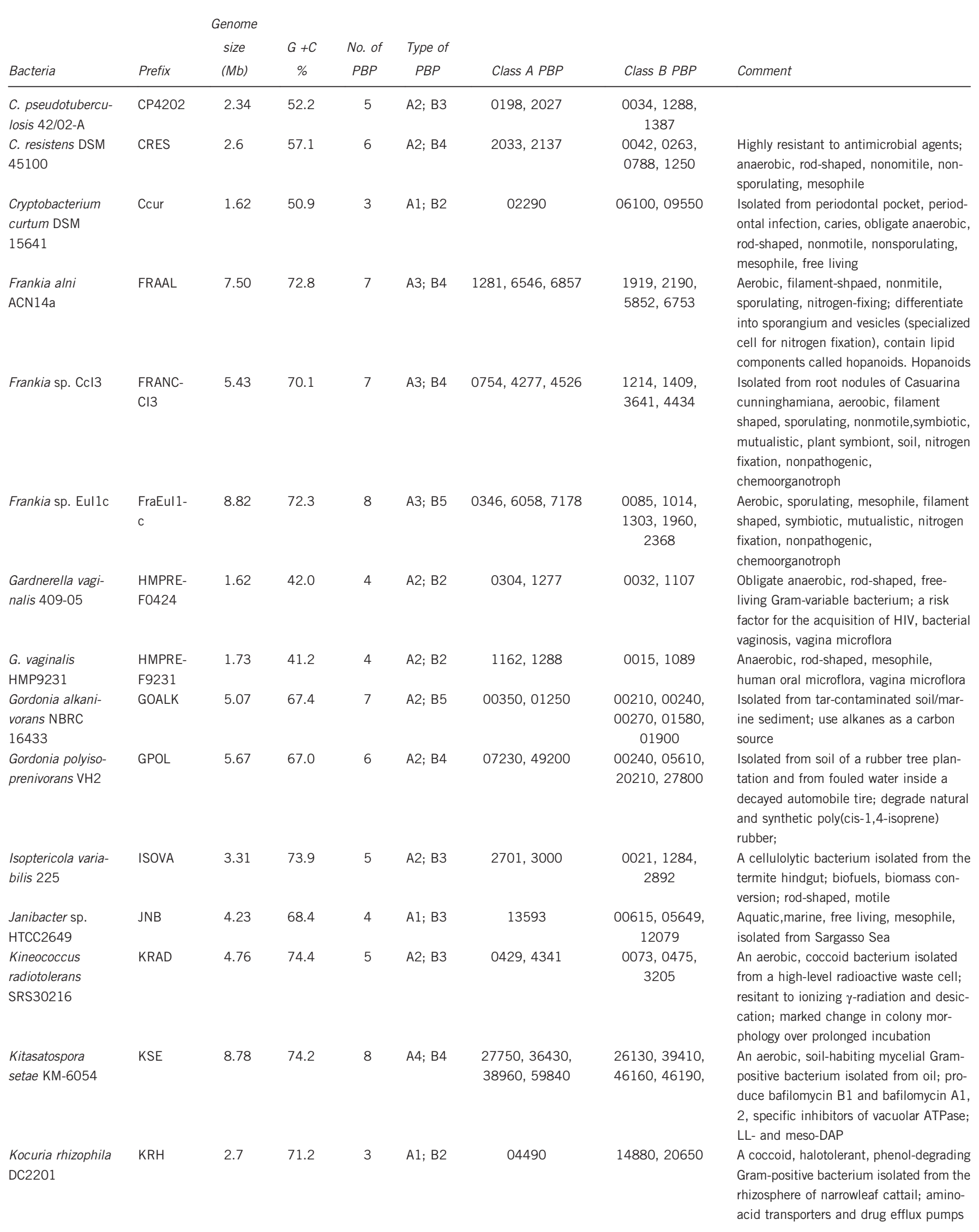




\begin{tabular}{|c|c|c|c|c|c|c|c|c|}
\hline Bacteria & Prefix & $\begin{array}{l}\text { Genome } \\
\text { size } \\
\text { (Mb) }\end{array}$ & $\begin{array}{c}G+C \\
\%\end{array}$ & $\begin{array}{l}\text { No. of } \\
\text { PBP }\end{array}$ & $\begin{array}{c}\text { Type of } \\
\text { PBP }\end{array}$ & Class A PBP & Class B PBP & Comment \\
\hline $\begin{array}{l}\text { Kytococcus seden- } \\
\text { tarius DSM } 20547\end{array}$ & KSED & 2.79 & 71.6 & 3 & B3 & & $\begin{array}{c}\text { 00200, } 09230 \\
16620\end{array}$ & $\begin{array}{l}\text { A free-living, nonmotile, human opportu- } \\
\text { nistic pathogen; grows as spherical/coc- } \\
\text { coid; produces monesin A and B; isolated } \\
\text { from varing environments such as human } \\
\text { skin and groundwater }\end{array}$ \\
\hline $\begin{array}{l}\text { Leifsonia xyli } \\
\text { subsp. xyli str. } \\
\text { CTCB07 }\end{array}$ & LXX & 2.58 & 67.7 & 6 & A4; B2 & $\begin{array}{l}0209003600 \\
05450,23190\end{array}$ & 00230,15320 & $\begin{array}{l}\text { An aerobic, rod-shaped, Gram-positive } \\
\text { bacterium, causes ratoon stunting disease } \\
\text { and affects sugarcane; a plant pathogen; } \\
\text { no endospore or motility; an unusual cell } \\
\text { wall peptidoglycan with 2,4-diaminobu- } \\
\text { tyric acid }\end{array}$ \\
\hline $\begin{array}{l}\text { Micromonospora } \\
\text { aurantiaca ATCC } \\
27029\end{array}$ & MICAU & 7.03 & 72.8 & 9 & A6; B3 & $\begin{array}{l}3350,4230 \\
4961,5144 \\
5927,6271\end{array}$ & $\begin{array}{l}0098,4478 \\
5070\end{array}$ & $\begin{array}{l}\text { Metabolic versatility like nitrogen fixation, } \\
\text { exhibit saphrophytic (living off dead tis- } \\
\text { sues) and symbiotic lifestyles, ubiquitous } \\
\text { in the environment, degrade plant cell } \\
\text { walls and fibers; spore formation; } \\
\text { bioremediation }\end{array}$ \\
\hline $\begin{array}{l}\text { Mobiluncus curtisii } \\
\text { ATCC } 43063\end{array}$ & $\begin{array}{l}\text { HMPRE- } \\
\text { F0573 }\end{array}$ & 2.15 & 55.4 & 4 & A2; B2 & 10708,10751 & 10644,11576 & $\begin{array}{l}\text { An anaerobic, rod shaped Gram-positive } \\
\text { bacterium found in the human vagina }\end{array}$ \\
\hline M. avium 104 & MAV & 5.48 & 69.0 & 5 & A2; B3 & 0071,0446 & $\begin{array}{l}0020,2330 \\
\quad 3723\end{array}$ & $\begin{array}{l}\text { M. avium complex causes a serious } \\
\text { infection in people with advanced AIDS; } \\
\text { mycolic acid-arabinoglactan- } \\
\text { peptidoglycan polymer form the cell wall }\end{array}$ \\
\hline $\begin{array}{l}\text { M. tuberculosis } \\
\text { H37Rv }\end{array}$ & RV & 4.41 & 65.6 & 5 & A2; B3 & 0050, 3682 & $\begin{array}{l}0016 \mathrm{c}, 2163 \mathrm{c} \\
2864 \mathrm{c}\end{array}$ & $\begin{array}{l}\text { Acid-fast, obligate aerobic, nonmotile, } \\
\text { rod-shaped bacterium; the causative } \\
\text { agent of tuberculosis; persists in a dor- } \\
\text { mant or latent form for years }\end{array}$ \\
\hline $\begin{array}{l}\text { Nakamurella mul- } \\
\text { tipartita DSM } \\
44233\end{array}$ & NAMU & 6.06 & 70.9 & 4 & A1; B3 & 0707 & $\begin{array}{l}0079,2190 \\
3930\end{array}$ & $\begin{array}{l}\text { An obligately aerobic chemoorgano- } \\
\text { trophic, polysaccharide-accumulating } \\
\text { Gram-positive bacterium isolated from } \\
\text { active sludge }\end{array}$ \\
\hline $\begin{array}{l}\text { Nocardia farcinica } \\
\text { IFM } 10152\end{array}$ & NFA & 6.02 & 70.8 & 8 & A3; B5 & $\begin{array}{l}03390,55490 \\
55570\end{array}$ & $\begin{array}{c}820,17600 \\
18430,41160 \\
54970\end{array}$ & $\begin{array}{l}\text { A Gram-positive, filamentous-growing soil } \\
\text { saprophyte, nocardiosis, mastitis, aerobic, } \\
\text { filament shaped, sporulating }\end{array}$ \\
\hline $\begin{array}{l}\text { Nocardioides sp. } \\
\text { JS614 }\end{array}$ & NOCA & 4.99 & 71.7 & 6 & A2; B4 & 0326,4676 & $\begin{array}{l}0024,3069 \\
3462,4600\end{array}$ & $\begin{array}{l}\text { An aerobic mesophillic Gram-positive } \\
\text { bacterium; grows on media containing } \\
\text { vinyl chloride and ethane; monooxygenase } \\
\text { system has a major role in the VC starva- } \\
\text { tion response }\end{array}$ \\
\hline $\begin{array}{l}\text { Nocardiopsis das- } \\
\text { sonvillei subsp. } \\
\text { dassonvillei DSM }\end{array}$ & Ndas & 5.77 & 72.8 & 5 & B5 & & $\begin{array}{l}0890,2552 \\
3385,3720 \\
5248\end{array}$ & $\begin{array}{l}\text { Aerobic, mesophile, free living, pulmonary } \\
\text { infection, actinomycetoma, lacks mycolic } \\
\text { acid }\end{array}$ \\
\hline
\end{tabular}


Table 1 (Continued)

\begin{tabular}{|c|c|c|c|c|c|c|c|c|}
\hline Bacteria & Prefix & $\begin{array}{c}\text { Genome } \\
\text { size } \\
(M b)\end{array}$ & $\begin{array}{c}G+C \\
\%\end{array}$ & $\begin{array}{l}\text { No. of } \\
\text { PBP }\end{array}$ & $\begin{array}{c}\text { Type of } \\
\text { PBP }\end{array}$ & Class A PBP & Class B PBP & Comment \\
\hline $\begin{array}{l}\text { P. acnes } \\
\text { KPA171202 }\end{array}$ & PPA & 2.56 & 60.0 & 4 & A2; B2 & 0126,2149 & 0185,0752 & $\begin{array}{l}\text { Anaerobic, rod-shaped, nonmotile, non- } \\
\text { sporulating, mesophile, pathogen, human } \\
\text { skin, acne }\end{array}$ \\
\hline P. acnes SK137 & $\begin{array}{l}\text { HMPRE- } \\
\text { F0675 }\end{array}$ & 2.5 & 60.1 & 4 & A2; B2 & 3139,5213 & 3226,3820 & $\begin{array}{l}\text { Anaerobic, nonspore forming, Gram- } \\
\text { positive bacterium; produces propionic } \\
\text { acid; non-toxigenic; a common resident of } \\
\text { the pilosebaceous (hair follicle) glands of } \\
\text { the human skin, acne vulgaris }\end{array}$ \\
\hline $\begin{array}{l}\text { Rothia dentocar- } \\
\text { iosa ATCC } 17931\end{array}$ & $\begin{array}{l}\text { HMRE- } \\
\text { F0733 }\end{array}$ & 2.51 & 53.7 & 3 & $\mathrm{~A} 1 ; \mathrm{B} 2$ & 10478 & 10948, 11665 & $\begin{array}{l}\text { Aerobic, pleomorphic, coccoid- to rod- } \\
\text { shaped bacterium frequently isolated } \\
\text { from the human oral cavity, periodontitis, } \\
\text { opportunistic infection, meningitis, } \\
\text { endocarditis }\end{array}$ \\
\hline $\begin{array}{l}\text { Rubrobacter xyla- } \\
\text { nophilus DSM } \\
9941\end{array}$ & Rxyl & 3.23 & 70.5 & 5 & A2; B3 & 1310,2308 & $\begin{array}{c}0022,1138 \\
1498\end{array}$ & $\begin{array}{l}\text { Isolated from thermally polluted industrial } \\
\text { runoff from a carpet factory, aerobic, rod- } \\
\text { shaped, nonmotile, nonsporulating, ther- } \\
\text { mophile, free living, radiation resistant, } \\
\text { nonpathogen, heterotroph, soil }\end{array}$ \\
\hline $\begin{array}{l}\text { Saccharomonos- } \\
\text { pora marina } \\
\text { XMU15 }\end{array}$ & SACMA & 5.97 & 68.9 & 5 & A2; B3 & 5053,5737 & $\begin{array}{l}0205,1393 \\
2176\end{array}$ & $\begin{array}{l}\text { Aerobic, Gram-positive bsacterium; forms } \\
\text { substrate and aerial mycelia, nonmotile; } \\
\text { isolated from ocean sediment; degrades } \\
\text { hemicellulose; contains an unusually } \\
\text { large number of glycosyltransferases }\end{array}$ \\
\hline $\begin{array}{l}\text { Saccharopolyspora } \\
\text { erythraea NRRL } \\
2338\end{array}$ & SACE & 8.21 & 71.1 & 7 & A4; B3 & $\begin{array}{l}0314,0385 \\
6352,7356\end{array}$ & $\begin{array}{l}0046,5864 \\
5990\end{array}$ & $\begin{array}{l}\text { Aerobic, filament-shpaed, nonmotile, } \\
\text { sporulating, free-living, Gram-positive } \\
\text { bacterium; produces erythromycin }\end{array}$ \\
\hline $\begin{array}{l}\text { Salinispora areni- } \\
\text { cola CNS-205 }\end{array}$ & SARE & 5.79 & 69.5 & 7 & A5; B2 & $\begin{array}{c}3240,3923 \\
4021,4796,5078\end{array}$ & 0051,3444 & $\begin{array}{l}\text { Isolated from tropical marine sediment; } \\
\text { produces staurosporine and nifamycin; } \\
\text { requires seawater for growth }\end{array}$ \\
\hline $\begin{array}{l}\text { Salinispora tropica } \\
\text { CNB-440 }\end{array}$ & STROP & 5.18 & 69.5 & 7 & A5; B2 & $\begin{array}{c}3015,3548, \\
3639,4354,4560\end{array}$ & 0046, 3218 & $\begin{array}{l}\text { Resides in ocean sediments; requires } \\
\text { seawater for growth; produces salinospor- } \\
\text { amide A, a potent inhibitor of the } 20 \mathrm{~S} \\
\text { proteasome; }\end{array}$ \\
\hline $\begin{array}{l}\text { Sanguibacter ked- } \\
\text { dieii DSM } 10542\end{array}$ & Sked & 4.25 & 71.9 & 3 & B3 & & $\begin{array}{c}00200,22860 \\
36830\end{array}$ & $\begin{array}{l}\text { Isolated from blood of dairy cows, rod- } \\
\text { shaped, nonsporulating, mesophile, free } \\
\text { living }\end{array}$ \\
\hline
\end{tabular}




\begin{tabular}{|c|c|c|c|c|c|c|c|c|}
\hline Bacteria & Prefix & $\begin{array}{c}\text { Genome } \\
\text { size } \\
(\mathrm{Mb})\end{array}$ & $\begin{array}{c}G+C \\
\%\end{array}$ & $\begin{array}{l}\text { No. of } \\
\text { PBP }\end{array}$ & $\begin{array}{c}\text { Type of } \\
\text { PBP }\end{array}$ & Class A PBP & Class B PBP & Comment \\
\hline $\begin{array}{l}\text { Slackia heliotrinir- } \\
\text { educens DSM } \\
20476\end{array}$ & SHEL & 3.17 & 60.2 & 3 & $\mathrm{~A} 1 ; \mathrm{B} 2$ & 08350 & 08800,14210 & $\begin{array}{l}\text { A nonmotile, obligate anaerobe, pyrrolizi- } \\
\text { dine alkaloids metabolizer Gram-positive } \\
\text { bacterium, isolated from the rumen of a } \\
\text { sheep }\end{array}$ \\
\hline $\begin{array}{l}\text { Stackebrandtia } \\
\text { nassauensis DSM } \\
44728\end{array}$ & Snas & 6.84 & 68.1 & 4 & A2; B2 & 1056,5713 & 2665,6473 & $\begin{array}{l}\text { An aerobic, nonmotile, degrade or hydro- } \\
\text { lyze allantoin, casein, aesculin, gelatin, } \\
\text { hypoxanthine, starch and tyrosine }\end{array}$ \\
\hline $\begin{array}{l}\text { S. albulus } \\
\text { CCRC11814 }\end{array}$ & K530 & 9.3 & 72.2 & 6 & $\mathrm{~A} 1 ; \mathrm{B} 5$ & 08126 & $\begin{array}{l}01507,21226 \\
23516,34418 \\
52485\end{array}$ & Production of poly-L-lysine \\
\hline S. albus J1074 & SSHG & 6.84 & 73.3 & 10 & A4; B6 & $\begin{array}{l}01811,02961 \\
03197,04158\end{array}$ & $\begin{array}{l}\text { 01149, } 01599 \\
02906,03834 \\
03835,04427\end{array}$ & $\begin{array}{l}\text { Nonmotile, aerobic, sporulating, Gram- } \\
\text { positive bacterium }\end{array}$ \\
\hline $\begin{array}{l}\text { S. avermitilis } \\
\text { MA-4680 }\end{array}$ & SAV & 9.03 & 70.7 & 13 & A6; B7 & $\begin{array}{l}3225,4294 \\
4423,4583 \\
5179,7219\end{array}$ & $\begin{array}{c}2952,3603 \\
3604,4339 \\
5458,6116 \\
6387\end{array}$ & $\begin{array}{l}\text { Soil and water Gram-positive filamentous } \\
\text { bacteria, produces avermectin, a human } \\
\text { and veterinary medicine }\end{array}$ \\
\hline $\begin{array}{l}\text { S. bingchenggen- } \\
\text { sis } B C W-1\end{array}$ & SBI & 11.94 & 70.8 & 12 & A6; B6 & $\begin{array}{l}\text { 03076, } 04174 \\
05361,05810 \\
06697,09068\end{array}$ & $\begin{array}{l}\text { 02283, } 04376 \\
05407,06233 \\
07119,07873\end{array}$ & $\begin{array}{l}\text { A soil bacterium; the largest bacterial } \\
\text { genome that has been sequenced to date; } \\
\text { produces milbemycin, an anthelmintic } \\
\text { macrolide }\end{array}$ \\
\hline $\begin{array}{l}\text { S.cattleya } \\
\text { NRRL8057 }\end{array}$ & SCAT & 6.28 & 72.9 & 11 & A4; B7 & $\begin{array}{l}1929,2889 \\
3140,3906\end{array}$ & $\begin{array}{l}0768,1207 \\
1730,1901 \\
3088,4153 \\
5676\end{array}$ & $\begin{array}{l}\text { Produces thienamyci, cephamycin } \mathrm{C} \text {, } \\
\text { penicillin } \mathrm{N} \text { and fluorinated metabolites }\end{array}$ \\
\hline $\begin{array}{l}\text { S. cattleya } \\
\text { DSM46488 }\end{array}$ & SCATT & 6.28 & 72.9 & 11 & A4; B7 & $\begin{array}{l}19200,28790 \\
31330,38910\end{array}$ & $\begin{array}{c}07700,12070 \\
17240,18950 \\
30790,41420 \\
56770\end{array}$ & \\
\hline $\begin{array}{l}\text { S. clavuligerus } \\
\text { ATCC27064 }\end{array}$ & SCLAV & 6.76 & 72.5 & 12 & A3; B9 & 2006, 2887, 3942 & $\begin{array}{c}1087,1301 \\
1774,2276 \\
2947,4154 \\
4179,4180 \\
4198\end{array}$ & $\begin{array}{l}\text { Clavulanic acid, cephamycin } \mathrm{C} \text {, deace- } \\
\text { toxycephalosporin } \mathrm{C} \text { and penicillin } \mathrm{N} \text {; } \\
\text { cannot use glucose as a carbon source, } \\
\text { because it lacks a glucose transport sys- } \\
\text { tem, has all the enzymes of the urea cycle }\end{array}$ \\
\hline $\begin{array}{l}\text { S. coelicoflavus } \\
\text { ZG0656 }\end{array}$ & SMCF & 8.48 & 71.8 & 11 & A3; B8 & $1708,4389,7595$ & $\begin{array}{l}3764,4686 \\
7469,7795 \\
7796,8190 \\
8286,8884\end{array}$ & $\begin{array}{l}\text { Produces novel acarviostatin family } \\
\alpha \text {-amylase inhibitors }\end{array}$ \\
\hline S. coelicolor $\mathrm{A} 3(2)$ & SCO & 8.67 & 72.1 & 13 & A4; B9 & $\begin{array}{l}2897,3580 \\
3901,5039\end{array}$ & $\begin{array}{c}1875,2090 \\
2608,3156 \\
3157,3771 \\
3847,4013 \\
5301\end{array}$ & $\begin{array}{l}\text { A filamentous, high } \mathrm{G}+\mathrm{C} \text { Gram-positive } \\
\text { bacterium; degrades chitin, takes part in } \\
\text { the nitrogen cycle }\end{array}$ \\
\hline S. collinus Tu365 & B446 & 8.27 & 72.6 & 9 & A3; B6 & $\begin{array}{c}15140,19060 \\
23580\end{array}$ & $\begin{array}{l}09755,10960 \\
13820,16355 \\
19320,24955\end{array}$ & Producer of kirromycin \\
\hline $\begin{array}{l}\text { S. davawensis } \\
\text { JCM4913 }\end{array}$ & BN159 & 9.47 & 70.6 & 11 & A4; B7 & $\begin{array}{l}3357,4150 \\
4546,5391\end{array}$ & $\begin{array}{l}3075,4478 \\
5121,5122 \\
5684,6352 \\
6632\end{array}$ & Producer of roseoflavin \\
\hline $\begin{array}{l}\text { S. flavogriseus } \\
\text { ATCC33331 }\end{array}$ & SFLA & 7.34 & 71.1 & 12 & A4; B8 & $\begin{array}{l}2228,3158 \\
3398,4003\end{array}$ & $\begin{array}{l}0559,1988 \\
3202,3620 \\
3741,4275 \\
4730,4938\end{array}$ & $\begin{array}{l}\text { An aerobic, Gram-positive bacterium iso- } \\
\text { lated from soil; produces cellulases and } \\
\text { xyanases }\end{array}$ \\
\hline $\begin{array}{l}\text { S. ghanaensis } \\
\text { ATCC } 14672\end{array}$ & SSFG & 8.22 & 72.2 & 10 & $\mathrm{~A} 4 ; \mathrm{B} 6$ & $\begin{array}{l}02387,02608 \\
03635,04479\end{array}$ & $\begin{array}{l}02394,03587 \\
04216,04217 \\
04765,05266\end{array}$ & $\begin{array}{l}\text { Aerobic, filamentous, nonmotile, high G } \\
+\mathrm{C} \text { Gram-positive bacterium, which pro- } \\
\text { duces moenomycin }\end{array}$ \\
\hline
\end{tabular}




\begin{tabular}{|c|c|c|c|c|c|c|c|c|}
\hline Bacteria & Prefix & $\begin{array}{c}\text { Genome } \\
\text { size } \\
(\mathrm{Mb})\end{array}$ & $\begin{array}{c}G+C \\
\%\end{array}$ & $\begin{array}{l}\text { No. of } \\
\text { PBP }\end{array}$ & $\begin{array}{l}\text { Type of } \\
\text { PBP }\end{array}$ & Class A PBP & Class B PBP & Comment \\
\hline $\begin{array}{l}\text { S. griseoaurantia- } \\
\text { cus M045 }\end{array}$ & SGM & 7.71 & 72.7 & 10 & A5; B5 & $\begin{array}{l}05501814,3549 \\
4216,6740\end{array}$ & $\begin{array}{l}0325,3502 \\
4547,5988 \\
5989\end{array}$ & $\begin{array}{l}\text { Isolated from marine sediment, produces } \\
\text { manumycin and chinikomycin }\end{array}$ \\
\hline $\begin{array}{l}\text { S. griseoflavus } \\
\text { Tu4000 }\end{array}$ & SSRG & 7.36 & 71.7 & 12 & A4; B8 & $\begin{array}{l}\text { 02182, } 02879 \\
03203,03961\end{array}$ & $\begin{array}{l}\text { 01957, 03076, } \\
03158,03705 \\
03706,04177 \\
04634,04850\end{array}$ & $\begin{array}{l}\text { Anaerobic, filamentous, nonmotile, free- } \\
\text { living, gram-positive bacterium }\end{array}$ \\
\hline $\begin{array}{l}\text { S. griseus subsp. } \\
\text { griseus NBRC } \\
13350\end{array}$ & SGR & 8.55 & 72.2 & 10 & A4; B6 & $\begin{array}{l}2494,3341 \\
3679,4647\end{array}$ & $\begin{array}{l}2203,3726 \\
4232,4340 \\
4934,5621\end{array}$ & $\begin{array}{l}\text { Anaerobic, filamentous, nonmotile, free- } \\
\text { living, Gram-positive bacterium; produces } \\
\text { streptomycin }\end{array}$ \\
\hline $\begin{array}{l}\text { S. hygroscopicus } \\
\text { subsp. jinggangen- } \\
\text { sis } 5008\end{array}$ & SHJG & 10.15 & 71.9 & 11 & A5; B6 & $\begin{array}{c}3853,4373 \\
5171,5432,6136\end{array}$ & $\begin{array}{l}3336,4100 \\
4627,4628 \\
5219,6411\end{array}$ & Produces validamycin; \\
\hline S. lividans TK24 & SSPG & 8.19 & 72.2 & 11 & A3; B8 & $\begin{array}{l}\text { 02649, } 03751 \\
04641\end{array}$ & $\begin{array}{l}\text { 02381, } 03670 \\
03808,03892 \\
04382,04383 \\
04919,05673\end{array}$ & $\begin{array}{l}\text { A Gram-positive, filamentous, soil } \\
\text { bacterium }\end{array}$ \\
\hline $\begin{array}{l}\text { S. pristinaespiralis } \\
\text { ATCC } 25486\end{array}$ & SSDG & 8.13 & 68.7 & 11 & A3; B8 & $\begin{array}{l}\text { 00591, 04208, } \\
\text { 06322, }\end{array}$ & $\begin{array}{l}02572,02766 \\
03053,06246 \\
06247,07051 \\
07138,07139\end{array}$ & $\begin{array}{l}\text { Aerobic, filamentous, nonmotile, Gram- } \\
\text { negative, sporulating bacterium; produces } \\
\text { pristinamycin }\end{array}$ \\
\hline $\begin{array}{l}\text { S. rimosus subsp. } \\
\text { rimosus } \\
\text { ATCC } 10970\end{array}$ & SRIM & 9.5 & 71.9 & 11 & A4; B7 & $\begin{array}{l}00295,08328 \\
13873,22689\end{array}$ & $\begin{array}{l}00065,04191 \\
06646,15770 \\
26297,31850 \\
31885\end{array}$ & $\begin{array}{l}\text { Bioconversion of quercetin into a novel } \\
\text { glycoside }\end{array}$ \\
\hline $\begin{array}{l}\text { S. roseosporus } \\
\text { NRRL } 11379\end{array}$ & $\begin{array}{l}\text { SROS- } \\
\text { N1_01- } \\
01000\end{array}$ & 7.76 & 71 & 10 & $\mathrm{~A} 4 ; \mathrm{B} 6$ & $\begin{array}{l}12023,17747 \\
19666,23998\end{array}$ & $\begin{array}{l}05995,09849 \\
13775,14509 \\
17500,25622\end{array}$ & $\begin{array}{l}\text { Aerobic, filamentous, nonmotile, Gram- } \\
\text { positive bacterium }\end{array}$ \\
\hline S. scabiei 87.22 & SCAB & 10.15 & 71.5 & 11 & A4; B7 & $\begin{array}{l}33601,41401 \\
56801,64431\end{array}$ & $\begin{array}{c}10101,29591 \\
45551,53611 \\
53621,60051 \\
70631\end{array}$ & $\begin{array}{l}\text { Aerobic, filamentous, nonmotile, sporu- } \\
\text { lating bacterium; an important plant } \\
\text { pathogen; produces phytotoxins called } \\
\text { thaxtomins: pathogenicity island }\end{array}$ \\
\hline $\begin{array}{l}\text { Streptomyces sp. } \\
\text { PAMC26508 }\end{array}$ & F750 & 7.53 & 71.1 & 10 & A4; B6 & $\begin{array}{l}2719,3337 \\
3596,4580\end{array}$ & $\begin{array}{l}1743,2434 \\
2998,3546 \\
4834,6320\end{array}$ & Production of clavaminate synthase 2 \\
\hline $\begin{array}{l}\text { Streptomyces sp. } \\
\text { SirexAA-E }\end{array}$ & SACTE & 7.41 & 71.7 & 11 & A4; B7 & $\begin{array}{l}2371,3027 \\
3329,4291\end{array}$ & $\begin{array}{l}1307,1519 \\
2029,2618 \\
2701,3283 \\
4532\end{array}$ & $\begin{array}{l}\text { Aerobic, filamentous, nonmotile, sporu- } \\
\text { lating free-living bacterium }\end{array}$ \\
\hline $\begin{array}{l}\text { S. sviceus ATCC } \\
29083\end{array}$ & SSEG & 9.31 & 69.8 & 10 & $\mathrm{~A} 4 ; \mathrm{B} 6$ & $\begin{array}{l}01073,07525 \\
03439,04164\end{array}$ & $\begin{array}{l}\text { 00010, } 00011 \\
00733,01896 \\
\text { 09019, } 09517\end{array}$ & $\begin{array}{l}\text { Aerobic, filamentous, nonmotile, sporu- } \\
\text { lating, free-living bacterium }\end{array}$ \\
\hline $\begin{array}{l}\text { S. tsukubaensis } \\
\text { NRRL18488 }\end{array}$ & STSU & 7.67 & 71.5 & 10 & A4; B6 & $\begin{array}{l}11540,15659 \\
19470,23388\end{array}$ & $\begin{array}{l}10421,17409 \\
21621,24378 \\
26744,27686\end{array}$ & Producer of tacrolimus (FK506) \\
\hline $\begin{array}{l}\text { S. venezuelae } \\
\text { ATCC } 10712\end{array}$ & SVEN & 8.23 & 72.4 & 10 & A4; B6 & $\begin{array}{l}2646,3350 \\
3677,4705\end{array}$ & $\begin{array}{l}1522,1745 \\
2386,2985 \\
3631,4995\end{array}$ & $\begin{array}{l}\text { Aerobic, filamentous, nonmotile, sporu- } \\
\text { lating free-living bacterium; produces } \\
\text { chloramphenicol, }\end{array}$ \\
\hline $\begin{array}{l}\text { S. violaceusniger } \\
\text { Tu4113 }\end{array}$ & STRVI & 10.66 & 71.0 & 11 & A5; B6 & $\begin{array}{c}1350,2314 \\
3845,8252,9005\end{array}$ & $\begin{array}{l}\text { 0275, 1135, } \\
3190,7171 \\
7897,7904\end{array}$ & $\begin{array}{l}\text { Aerobic, filamentous, motile, sporulating, } \\
\text { mesophilic, bacterium; produces spiro- } \\
\text { fungin, antifungal agent }\end{array}$ \\
\hline $\begin{array}{l}\text { S. viridochromo- } \\
\text { genes DSM } 40736\end{array}$ & SSQG & 8.55 & 71.1 & 11 & A5; B6 & $\begin{array}{l}\text { 02328, } 02941 \\
03901,04279 \\
05113\end{array}$ & $\begin{array}{l}01781,02628 \\
03242,03243 \\
03958,05348\end{array}$ & $\begin{array}{l}\text { Aerobic, filamentous, nonmotile, sporu- } \\
\text { lating bacterium }\end{array}$ \\
\hline $\begin{array}{l}\text { S. zinciresistens } \\
\mathrm{K} 42\end{array}$ & SZN & 8.22 & 72.5 & 10 & $\mathrm{~A} 4 ; \mathrm{B} 6$ & $\begin{array}{l}06389,16730 \\
18682,28493\end{array}$ & $\begin{array}{l}02952,10458 \\
13352,17932 \\
18819,22026\end{array}$ & $\begin{array}{l}\text { Aerobic, filamentous, nonmotile, non- } \\
\text { sporulating, halophilic bacterium; iso- } \\
\text { lated from soil from a zinc and copper } \\
\text { mine }\end{array}$ \\
\hline
\end{tabular}




\begin{tabular}{|c|c|c|c|c|c|c|c|c|}
\hline Bacteria & Prefix & $\begin{array}{c}\text { Genome } \\
\text { size } \\
(\mathrm{Mb})\end{array}$ & $\begin{array}{c}G+C \\
\%\end{array}$ & $\begin{array}{l}\text { No. of } \\
\text { PBP }\end{array}$ & $\begin{array}{c}\text { Type of } \\
\text { PBP }\end{array}$ & Class A PBP & Class B PBP & Comment \\
\hline $\begin{array}{l}\text { Streptosporangium } \\
\text { roseum DSM } \\
43021\end{array}$ & SROS & 10.34 & 70.9 & 11 & A4; B7 & $\begin{array}{l}2902,3010 \\
8177,9363\end{array}$ & $\begin{array}{l}0113,1441 \\
1456,2864 \\
3583,4062 \\
7683\end{array}$ & $\begin{array}{l}\text { Aerobic, filamentous, nonmotile, sporu- } \\
\text { lating, mesophilic bacterium; produces } \\
\text { angucycline, an inhibitor of the } \\
\text { endothelin-converting enzyme }\end{array}$ \\
\hline $\begin{array}{l}\text { Thermobifida } \\
\text { fusca YX }\end{array}$ & TFU & 3.64 & 67.5 & 4 & A2; B2 & 0570, 3097 & 1416,2475 & $\begin{array}{l}\text { Aerobic, rod-shaped, nonmotile, sporulat- } \\
\text { ing, moderate thermophilic soil bacter- } \\
\text { ium; degrades organic material and } \\
\text { dcllulose; farmer's lung, mushroom work- } \\
\text { er's disease, respiratory infection }\end{array}$ \\
\hline $\begin{array}{l}\text { Thermobispora } \\
\text { bispora DSM } \\
43833\end{array}$ & TBIS & 4.19 & 72.4 & 10 & A4; B6 & $\begin{array}{l}0195,1426 \\
3106,3566\end{array}$ & $\begin{array}{l}0053,0796 \\
1401,1685 \\
1727,2465\end{array}$ & $\begin{array}{l}\text { Aerobic, filamentous, nonmotile, sporu- } \\
\text { lating, thermophilic bcterium }\end{array}$ \\
\hline $\begin{array}{l}\text { T. curvata DSM } \\
43183\end{array}$ & TCUR & 5.64 & 71.6 & 8 & A4; B4 & $\begin{array}{l}1026,1268 \\
4921,4955\end{array}$ & $\begin{array}{l}0065,1542 \\
2932,4002\end{array}$ & $\begin{array}{l}\text { An aerobic, cellulolytic, thermophilic } \\
\text { Gram-positive bacterium; produces a } \\
\text { number of industrially important com- } \\
\text { pounds like cellulase, alpha-amylase and } \\
\text { polygalacturonate lyase }\end{array}$ \\
\hline $\begin{array}{l}\text { Tropheryma whip- } \\
\text { plei str. Twist }\end{array}$ & TWT & 0.93 & 46.3 & 3 & $\mathrm{~A} 1 ; \mathrm{B} 2$ & 0705 & 0222,0776 & $\begin{array}{l}\text { Aerobic, rod-shaped, nonmotile, nonspor- } \\
\text { ulating, mesophilic bacterium; deficient } \\
\text { in amino acid metabolisms, the lack of } \\
\text { clear thioredoxin and thioredoxin reduc- } \\
\text { tas; causes Whipple's disease }\end{array}$ \\
\hline $\begin{array}{l}\text { Tropheryma whip- } \\
\text { plei Tw08/27 }\end{array}$ & TW & 0.93 & 46.3 & 3 & $\mathrm{~A} 1 ; \mathrm{B} 2$ & 0722 & 0548,0787 & $\begin{array}{l}\text { A Gram-positive, filamentous, aerobic, } \\
\text { soil-dwelling actinomycete. It is the cau- } \\
\text { sative agent of the Whipple's disease }\end{array}$ \\
\hline $\begin{array}{l}\text { Tsukamurella } \\
\text { paurometabola } \\
\text { DSM } 20162\end{array}$ & TPAU & 4.38 & 68.4 & 7 & A2; B5 & 3939, 4192 & $\begin{array}{l}0029,0349 \\
1690,2652 \\
3973\end{array}$ & $\begin{array}{l}\text { Obligate aerobic, rod-shaped, nonomotile, } \\
\text { nonsporulating, mesophilic bacterium; } \\
\text { isolated from ovaries of Cimex lectularius; } \\
\text { a human opportunistic pathogen }\end{array}$ \\
\hline
\end{tabular}

Abbreviations: $G+C$, guanine+cytosine; $P B P$, penicillin-binding protein.

Catenulispora, Renibacterium, Arthrobacter, Saccharomonospora and Micromonospora, constitute cluster IV. Some PBPs of Actinomyces and Mobiluncus fall into subcluster IV-2. PBP members that from suborder Micrococcineae are partly overlapped and distributed in the clusters IV and V. Kytococcus, Isoptericola, Cellulomonas, Sanguibacter, Beutenbergia, Janibacter, Renibacterium and Arthrobacter are members of suborder Micrococcineae. PBPs of order Bifidobacteriales form subcluster III. The cluster V includes PBPs of suborder Micrococcineae members such as Clavibacter, Leifsonia, Tropheryma, Micrococcus, Rothia, Kocuria, Renibacterium and Arthorobacter. However, PBPs of suborder Micrococcineae are also distributed in the cluster IV, as described above. No PBPs of Micrococcineae make a phylogenetically distinct, coherent cluster and are dispersed in clusters IV and $V^{4}$ The subclusters VI-1, VI-2 and VI-3 consist of PBPs of suborder Corynebacterineae (Corynebacterium, Tsukamurella, Gordonia, Mycobacterium, Rhodococcus, Nocardia and Amycolicicoccus), suborder Pseudonocardineae (Saccharopolyspora, Saccharomonospora and Actinosynnema) and suborder Frankineae (Nakamurella). However, PBPs of Corynebacterineae and Pseudonocardineae compose different branches in the subclusters. The suborders Corynebacterineae and Pseudonocardineae are closely related taxonomically ${ }^{4,22}$ and, in addition, Nakamurella multipartite, which is currently a member of suborder Frankineae, is proposed to be closely related to Pseudonocardineae. ${ }^{4}$
The PBPs of most Frankia species belong to subclusters VII-1, VII-2, VII-4 and VII-5 which contain also PBPs of suborder Frankineae (Frankia and Acidothermus), suborder Micromonosporineae (Salinispora, Micromonospora and Actinoplanes) and suborder Glycomycineae (Stackebrandtia).

As described above, Streptomyces species carry more PBPs than other species. Reflecting this fact, PBPs of Streptomyces species form seven large subclusters of VIII-1, VIII-2, VIII-3, VIII-4, VIII-5, VIII-6 and VIII-7. SCAB_10101 possesses neither essential serine nor lysine residues which are involved in the enzymatic catalysis (the amino-acid sequence in this region is Thr-Thr-Phe-Ser), so that it is excluded from further analysis.

Intriguingly, more than half of Streptomyces species hold two successive class B PBPs (Figure 1 and Table 1). For example, SSHG_03834 and SSHG_03835 (a similarity value E is $1.2 \mathrm{e}-62$, the same hereafter), SAV_3603 and SAV_3604 (3e-68), SCLAV_4179 and SCLAV_4180 $(1.5 \mathrm{e}-11), \quad$ SMCF_7795 and SMCF_7796 $(1.1 \mathrm{e}-48), \mathrm{SCO} 3156$ and SCO3157 $(2.8 \mathrm{e}-47), \mathrm{BN} 159 \_5121$ and BN159_5122 (3.4e-65), SSFG_04216 and SSFG_04217 (1.3e-56), SGM_5988 and SGM_5989 (1.3e-58), SSRG_03705 and SSRG_03706 (3.2e-62), SHJG_4627 and SHJG_4628 (8.6e-62), SSPG_04382 and SSPG_04383 (2.7e-59), SSDG_07138 (125aa) and SSDG_07139 (too short to compare), SCAB_53611 and SCAB_53621 


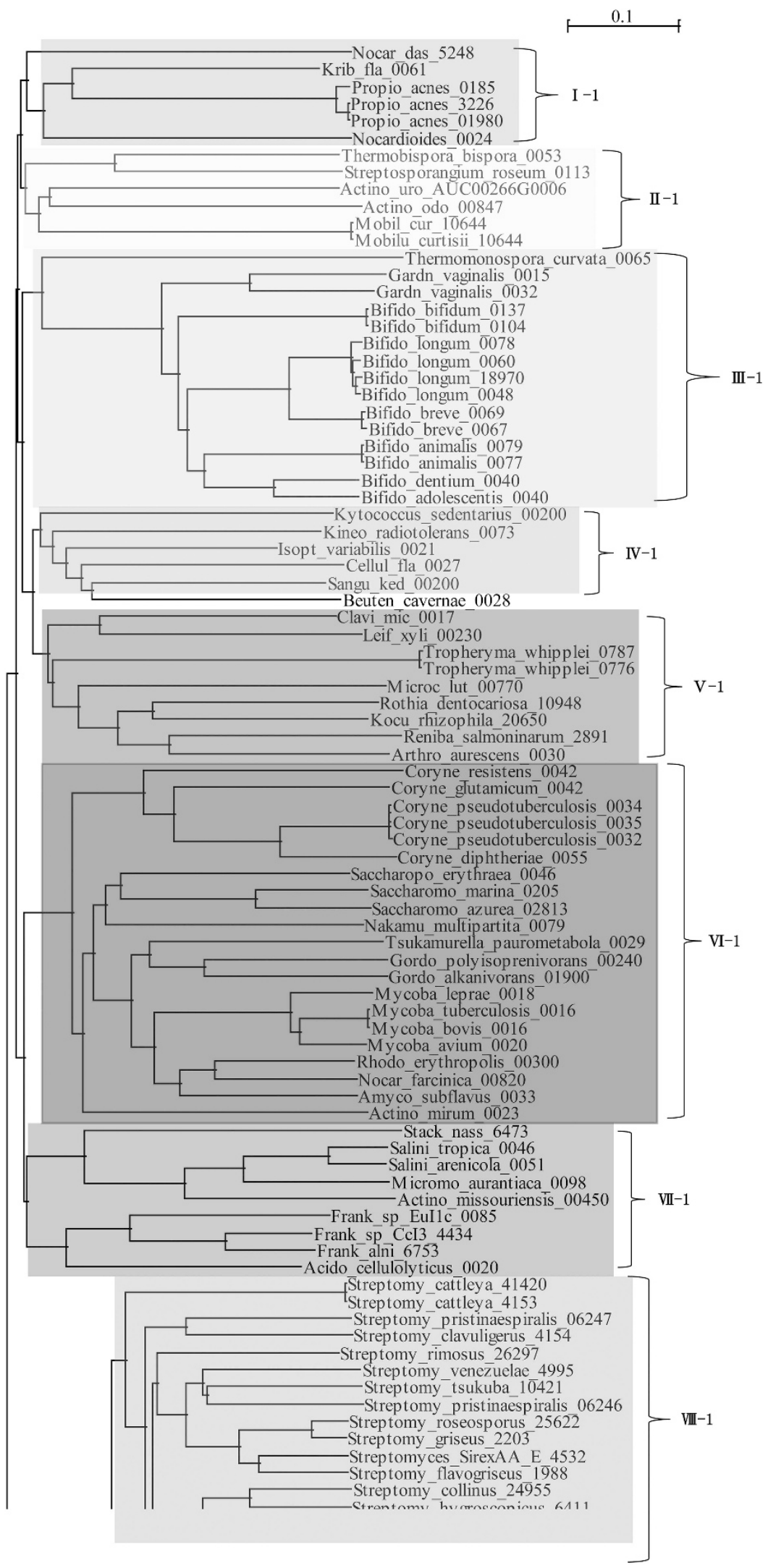

Figure 1 Phylogenetic tree of 446 class B PBPs listed in Table 1 from Actinobacteria. The tree was constructed by using ClustalX $2^{40}$ as SCO4049 (penicillin acylase) as outgroup. A full color version of this figure is available at The Journal of Antibiotics journal online. 


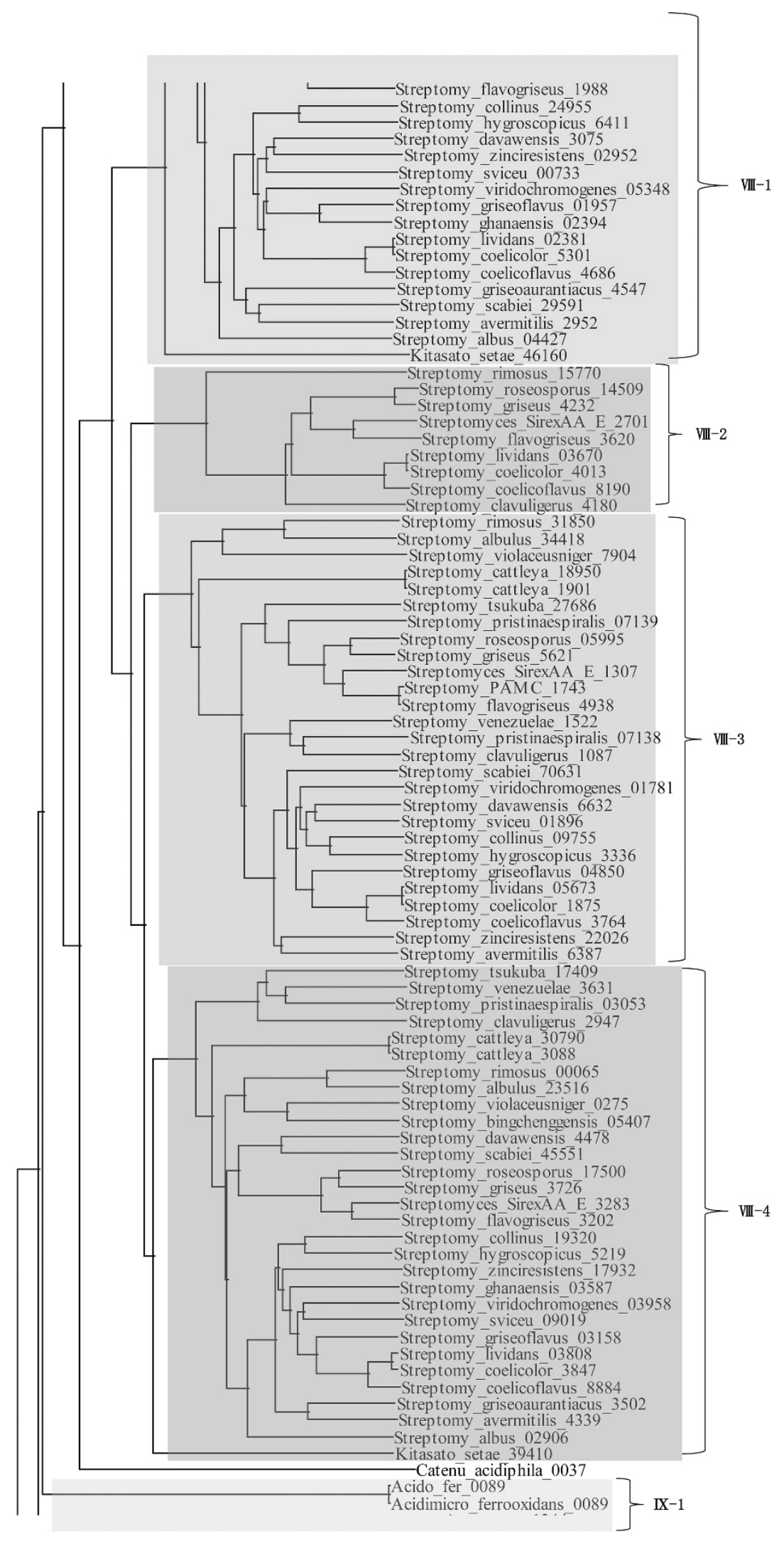

Figure 1 Continued. 


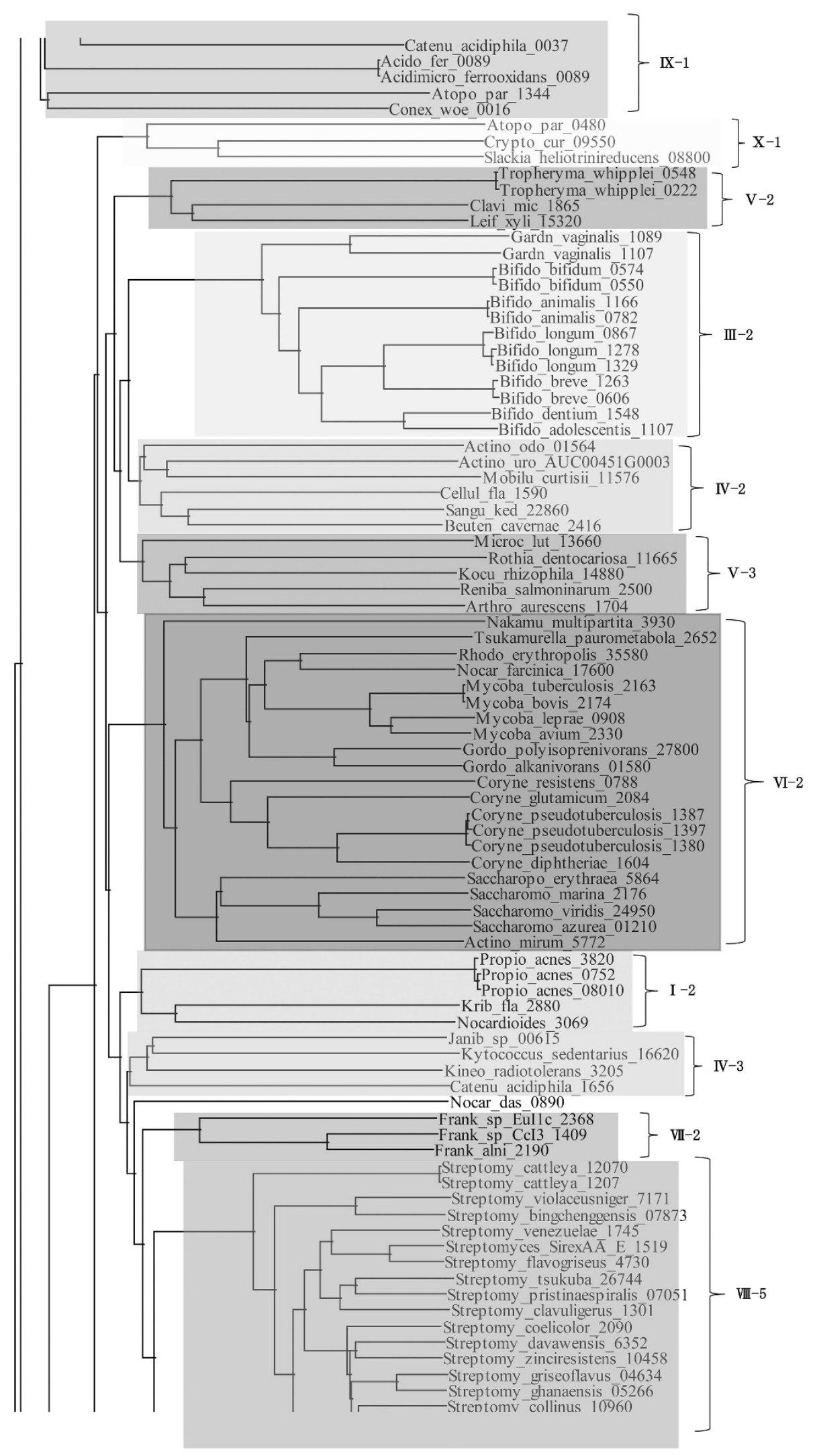

Figure 1 Continued. 


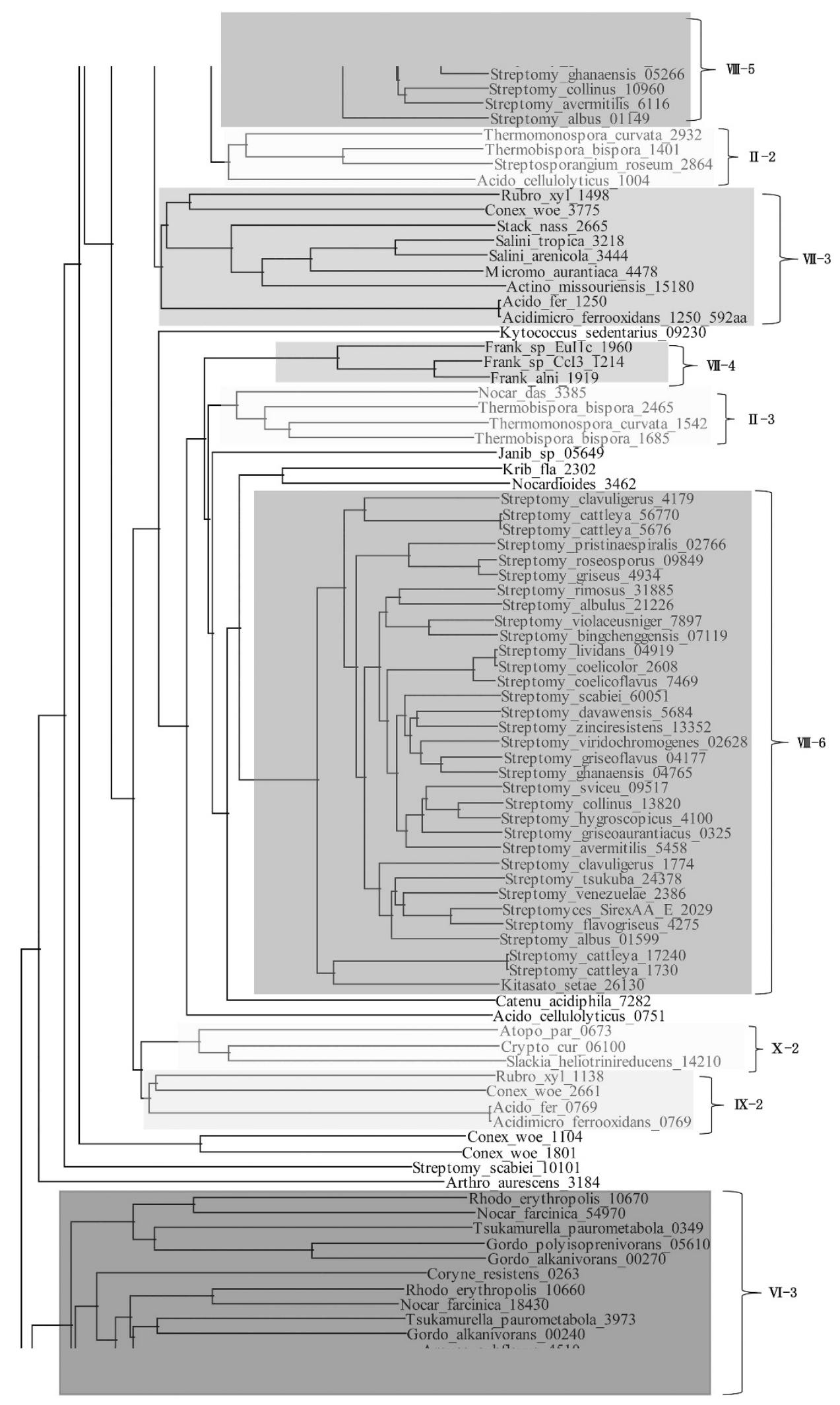

Figure 1 Continued. 


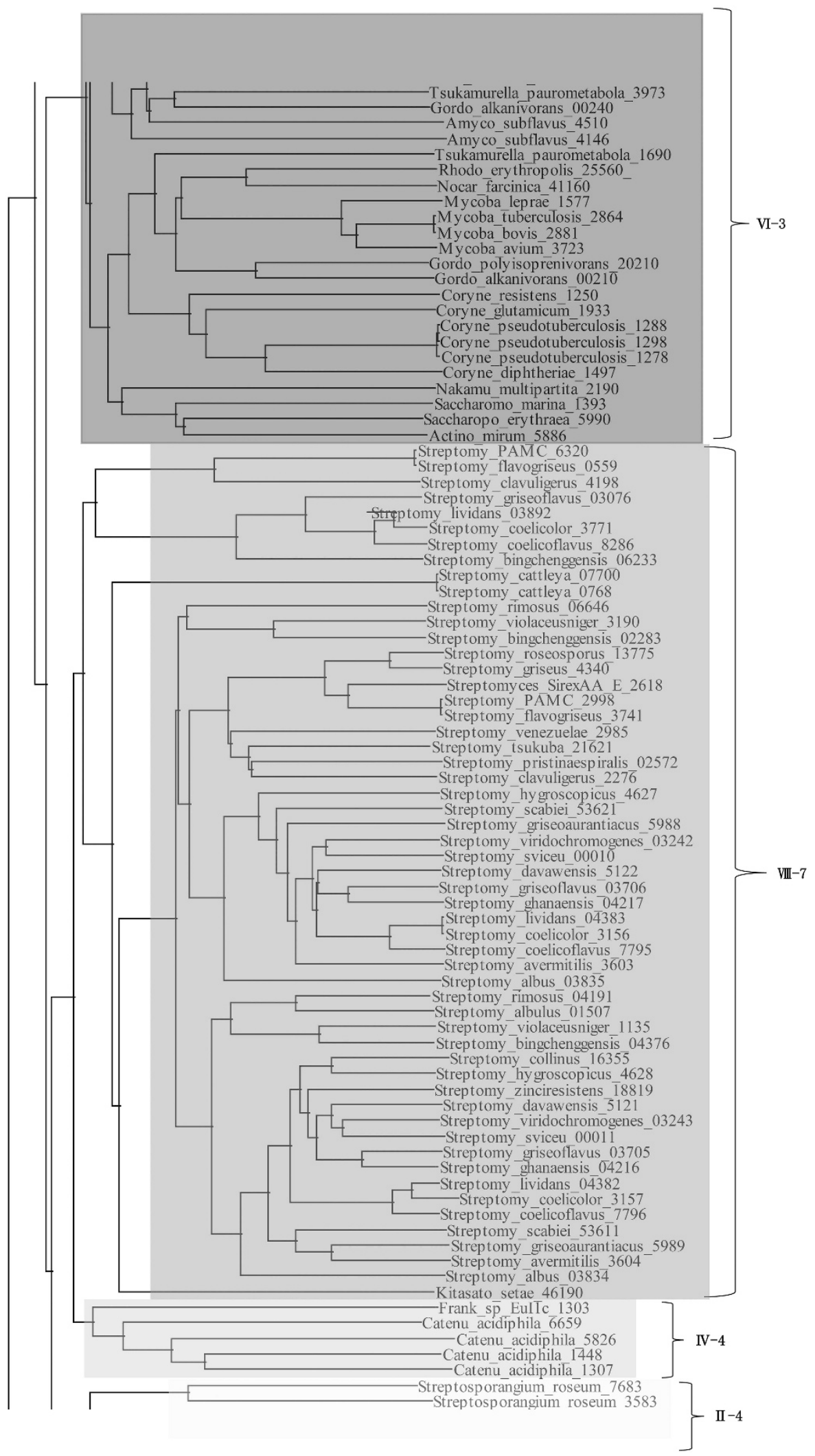

Figure 1 Continued. 


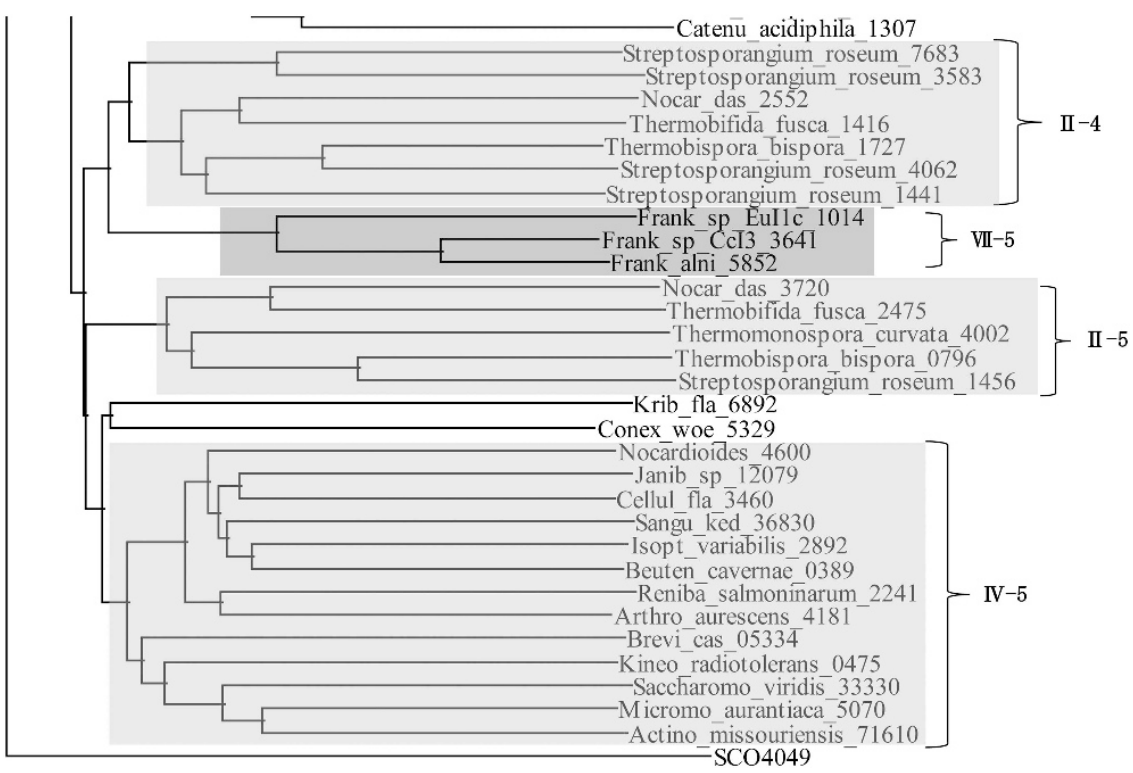

Figure 1 Continued.

Table 2 Comparison of genomic arrangements adjacent to PBPs in three Streptomyces species

\begin{tabular}{|c|c|c|c|c|c|c|c|c|}
\hline \multicolumn{3}{|c|}{ S. clavuligerus (SCLAV) } & \multicolumn{3}{|c|}{ S. cattleya (SCAT) } & \multicolumn{3}{|c|}{ S. coelicolor (SCO) } \\
\hline Gene no. & No. of aa & Function & Gene no. & No. of aa & Function & Gene no. & No. of aa & Function \\
\hline 1769 & 1456 & Ribonuclease E & 1725 & 136 & Hypothetical protein & 2603 & 518 & Integrase \\
\hline 1770 & 251 & Radical SAM protein & 1726 & 148 & Hypothetical protein & 2604 & 311 & Hypothetical protein \\
\hline 1771 & 651 & Fe-S oxidoredactase & 1727 & 61 & Hypothetical protein & 2605 & 233 & Hypothetical protein \\
\hline 1772 & 520 & Metal-binding protein & 1728 & 651 & Hypothetical protein & 2606 & 661 & Hypothetical protein \\
\hline 1773 & 401 & RodA, FtsW & 1729 & 401 & Hypothetical protein & 2607 & 398 & Sfr protin \\
\hline 1774 & 773 & Penicillin-binding protein & 1730 & 738 & Penicillin-binding protein & 2608 & 769 & Penicillin-binding protein \\
\hline 1775 & 219 & MreD & 1731 & 209 & MreD & 2609 & 223 & MreD \\
\hline 1776 & 372 & MreC & 1732 & 302 & MreC & 2610 & 341 & MreC \\
\hline 1777 & 343 & MreB, Mbl & 1733 & 343 & MreB & 2611 & 343 & MreB \\
\hline 1778 & 144 & Nucloside diphosphate kinase & 1734 & 137 & Nucloside diphosphate kinase & 2612 & 137 & Nucloside diphosphate kinase \\
\hline 1779 & 121 & Membrane protein & 1735 & 122 & Hypothetical protein & 2613 & 118 & Hypothetical protein \\
\hline 1780 & 505 & Dihydrofolate synthase & 1736 & 510 & Tetrahydrofolate synthase & 2614 & 444 & Tetrahydrofolate synthase \\
\hline 1781 & 417 & Two component histidine kinase & 1737 & 877 & Valyl-tRNA synthetase & 2615 & 874 & Valyl-tRNA synthetase \\
\hline 1782 & 875 & Valyl-tRNA synthetase & 1738 & 284 & Hypothetical protein & 2616 & 335 & Hypothetical protein \\
\hline 1783 & 386 & PAT1 multi-domain protein & 1739 & 427 & ATP-dependent protease & 2617 & 428 & ATP-dependent protease \\
\hline 1784 & 464 & ATP-dependent protease & & & & & & \\
\hline
\end{tabular}

A full color version of this table is available at The Journal of Antibiotics journal online.

$(3.2 \mathrm{e}-59), \quad$ SSEG_00010 and SSEG_00011 (9.3e-61), and SSQG_03242 and SSQG_03243 (3.5e-61). The amino-acid sequences of these pairs of PBPs are not only very similar to each other, but also all the sequences are closely related and pertain to the subcluster VIII-7 in the phylogenetic tree (Figure 1). That is, the amino-acid sequence identity and similarity of PBPs in subcluster VIII-7 are in the range of $49.2-51.8 \%$ and $71.8-77.8 \%$, respectively. Furthermore, the nucleotide sequences of each pair are arrayed in the same direction, indicating that they were duplicated and transferred to each other very recently. The pair of S. clavuligerus SCLAV_4179 and SCLAV_4180 is an exception. S. clavuligerus is a cephamycin and clavulanic acid producer. These PBPs of $S$. clavuligerus belong to the different subclusters (VIII-6 and VIII-2) and the similarity of the amino-acid sequences is very low (the E-value is 1.5e-11). Although
S. clavuligerus possesses two PBPs in subcluster VIII-7, SCLAV_2276 and SCLAV_4198, their similarity value $\mathrm{E}$ is not so low (3.1e-28). In addition, $S$. cattleya, a cephamycin and thienamycin producer, carries only one PBP (SCAT_0768) in this subcluster. This peculiar behavior may be related to $\beta$-lactam production. The two PBPs of S. clavuligerus SCLAV_4179 and SCLAV_4180 are located at the end of cephamycin-clavulanic acid biosynthetic gene cluster, but arrayed in the reverse direction. Moreover, PBP SCLAV_4179 in S. clavuligerus is reported to have a low affinity to $\beta$-lactam antibiotics and is essential to the growth, ${ }^{23}$ consequently it is presumed to be involved in the selfresistance. Interestingly, the amino-acid sequence of SCLAV_4179 is highly similar to that of SCAT_5676 (the similarity value E is $8.4 \mathrm{e}-186)$ of $S$. cattleya, indicating that the PBP genes were interchanged between the two species as a whole-cephamycin biosynthetic 


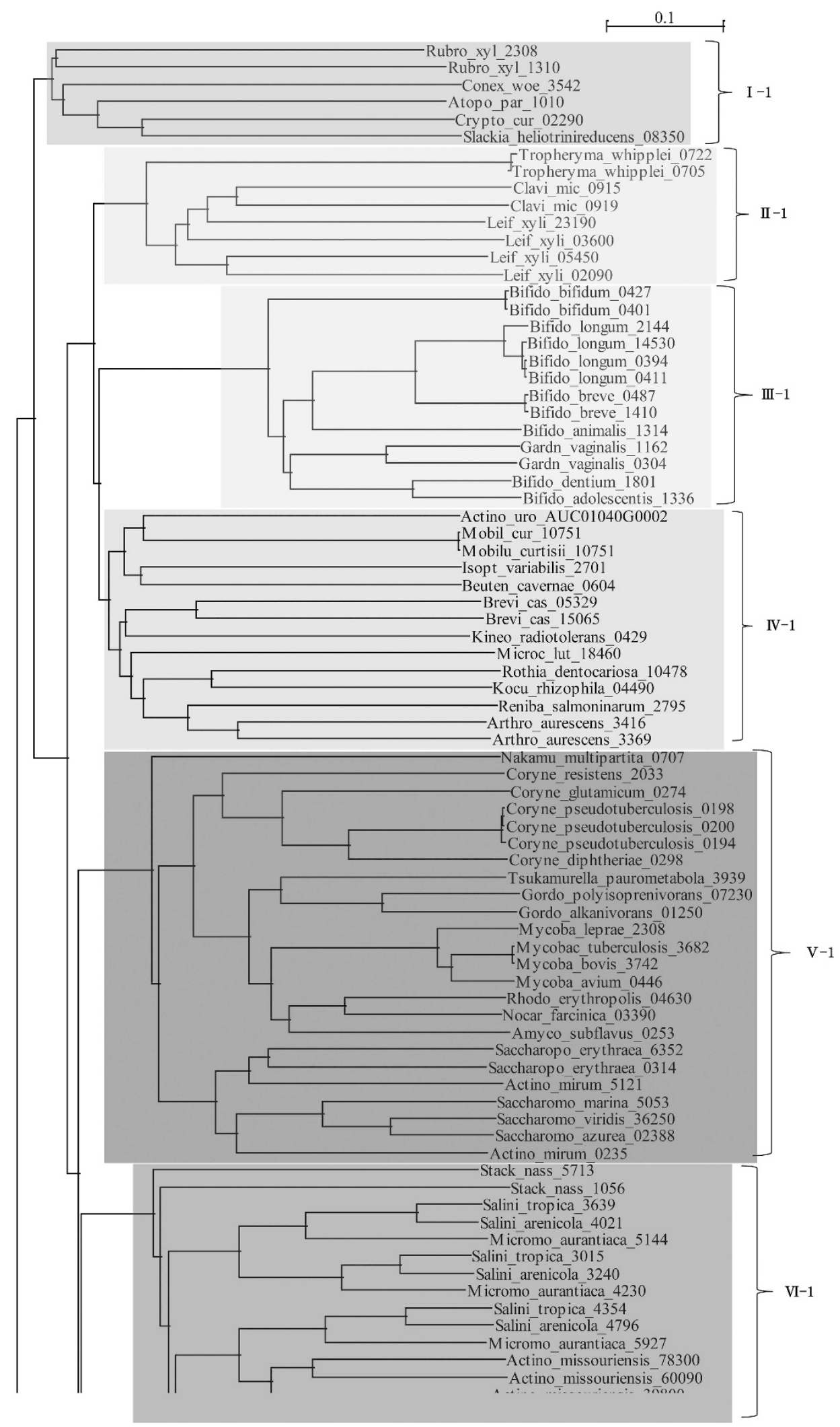

Figure 2 Phylogenetic tree of 292 class A PBPs listed in Table 1 from Actinobacteria. The tree was constructed by using ClustalX $2^{40}$ as SCO4049 (penicillin acylase) as outgroup. A full color version of this figure is available at The Journal of Antibiotics journal online. 


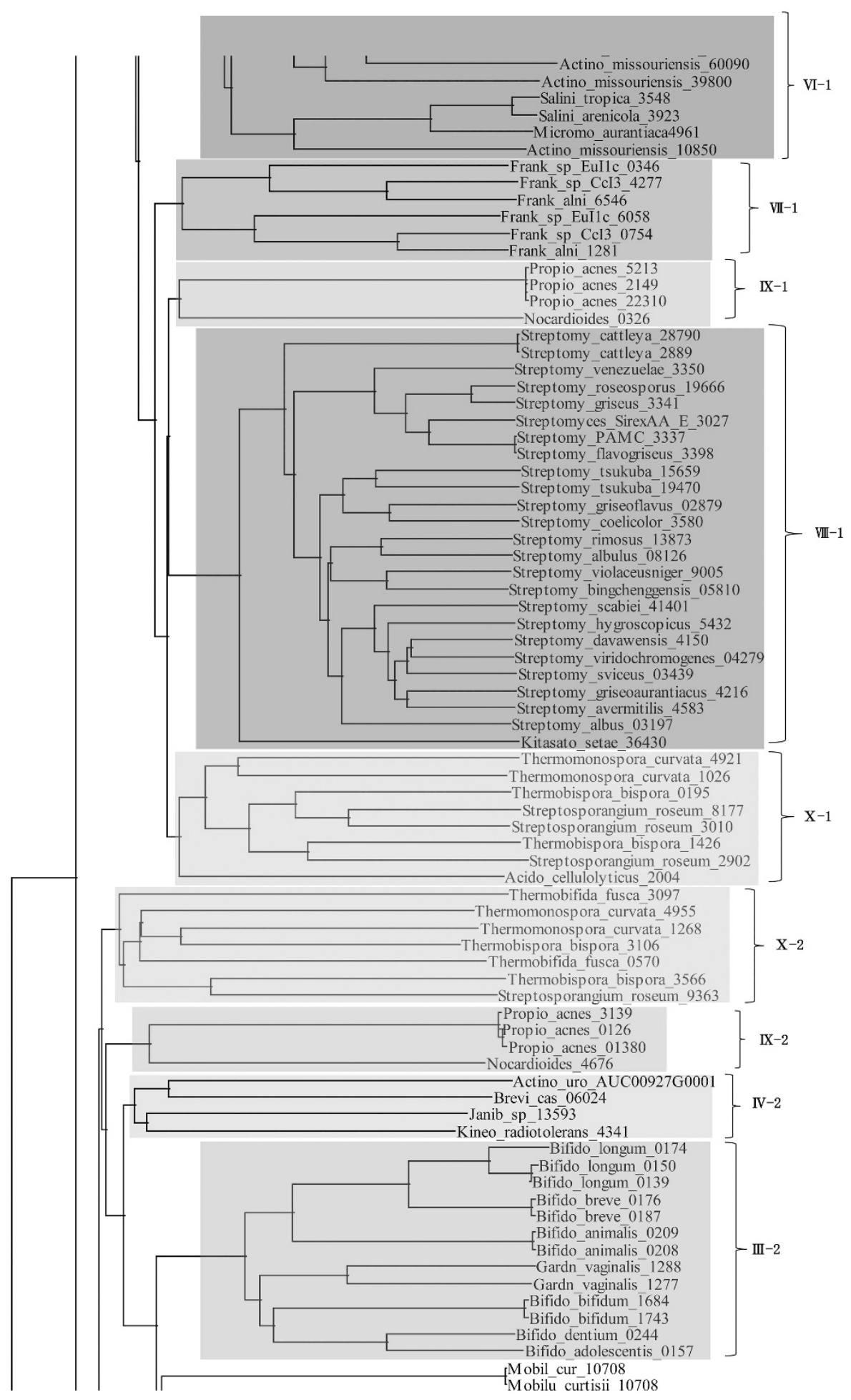

Figure 2 Continued.

gene cluster, and the clavulanic acid gene cluster was inserted in this region later. In $S$. cattleya, the protein of the similar amino-acid sequence to SCLAV_4180 is located not in the next to SCAT_5676 but in the completely different position as SCAT_3088, where no $\beta$-lactam biosynthetic gene is present. On the other hand, two proteins having highly similar amino-acid sequences to SCLAV_4179 (SCAT_5676 and SCAT_1730, the similarity E-values are 8.4e-186 and 1e-144, respectively) exist in $S$. cattleya. In $S$. clavuligerus, a similar PBP to SCLAV_4179 is present as SCLAV_1774 (E-value is 2.9e-159). Furthermore, a similar protein to SCLAV_4179 is also found in S. coelicolor (SCO2608, an E-value is 1.1e-160). Comparison of the genomic arrangements in these three species reveals similar arrangements of the genes, at least in the downstream of PBPs (Table 2). Moreover, similar proteins to SCLAV_4179 are present not only in 


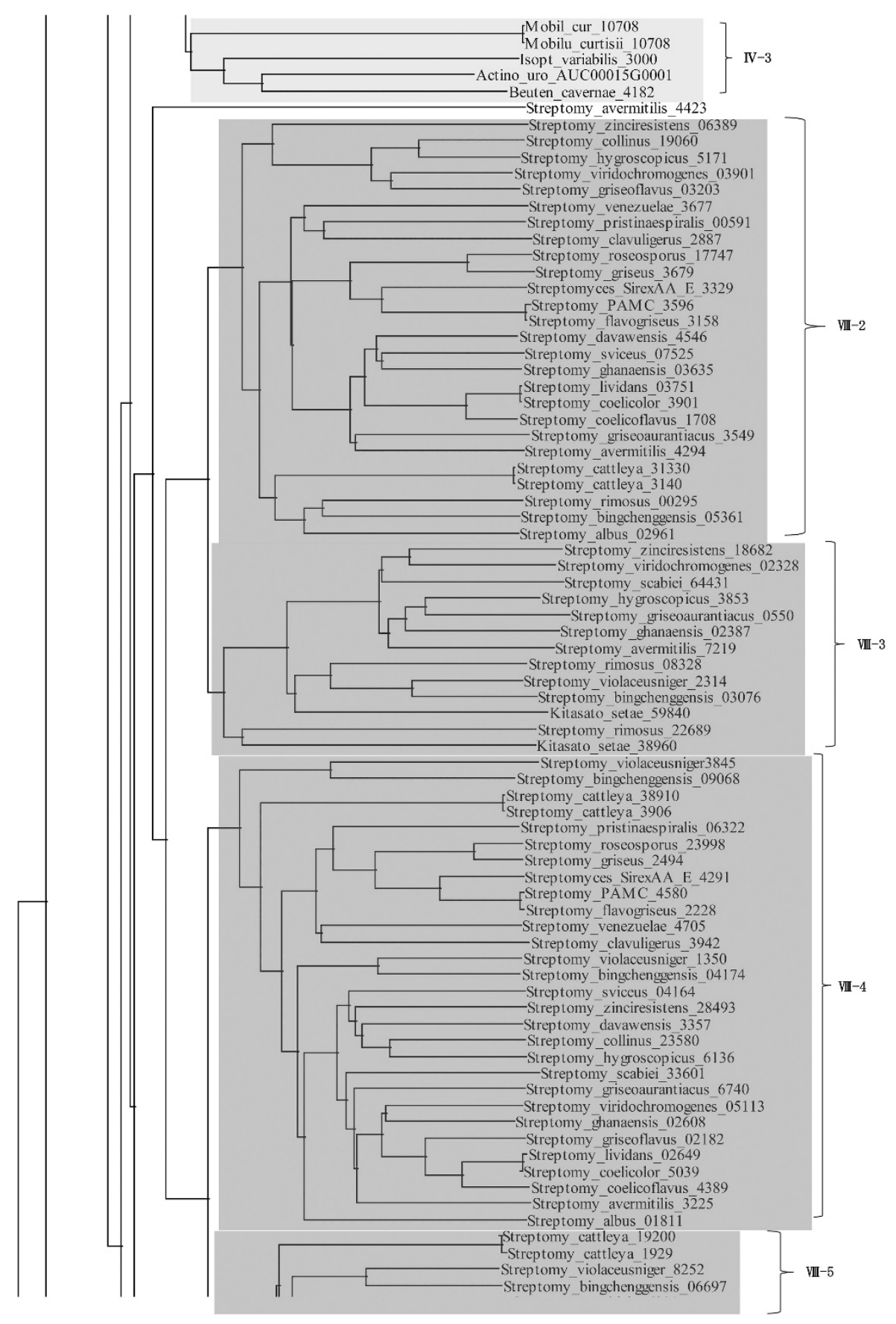

Figure 2 Continued.

Streptomyces species such as S. avermitilis MA-4680 (SAV_5458, an E-value is $2.5 \mathrm{e}-158$, the same hereafter), $S$. lividans TK24 (SSPG_04919, 3.7e-169), S. viridochromogenes DSM 40736 (SSQG_02628, 5.6e-161), S. scabiei 87.22 (SCAB_60051, 1.7e-174), S. griseus (SGR_4934, 4.5e-165) and S. hygroscopicus (SHJG_4100, $2.9 \mathrm{e}-163$ ), but also in Catenulispora acidiphila DSM 44928 (Caci_7282, 1.2e-101), Kitasatospora setae KM-6054 (KSE_26130, 5.9e-137), Kribbella flavida DSM 17836 (Kfla_2302, 4.3e-105) and Nocardioides sp. JS614 (Noca_3462, 2.0e -108). ${ }^{24}$ That is, the amino-acid sequence identity and similarity are in the range of $74.3-75.5 \%$ and $90.8-92.5 \%$ in Streptomyces, respectively, and $45.7-62.7 \%$ and $72.6-83.2 \%$ in other species, respectively. None of these species is reported to produce $\beta$-lactam antibiotics, suggesting that SCLAV_4179 and its analogs in Streptomyces at least are not related to $\beta$-lactam biosynthesis but associated only with $\beta$-lactam resistance. This is supported by the fact that similar proteins to SCLAV_4179 are also found in other Actinobacteria, such as Thermomonospora curvata DSM 43183 (Tcur_1542, 6.3e-99), Frankia sp. EuI1c (FraEuI1c_1960, 4.9e-76), Nocardiopsis dassonvillei (Ndas_3385, 5.2e-108) and Janibacter sp. HTCC2649 (JNB_05649, $4.3 \mathrm{e}-72), \quad \beta$-proteobacteria, such as Methylobacillus flagellatus KT (YP_546600, 5.2e-40) and Janthinobacterium lividum (WP_010393193, 3.9e-49), and $\gamma$-proteobacteria, such as Plesiomonas shigelloides (WP_010864271, 9.8e-36) and Pseudomonas putida 


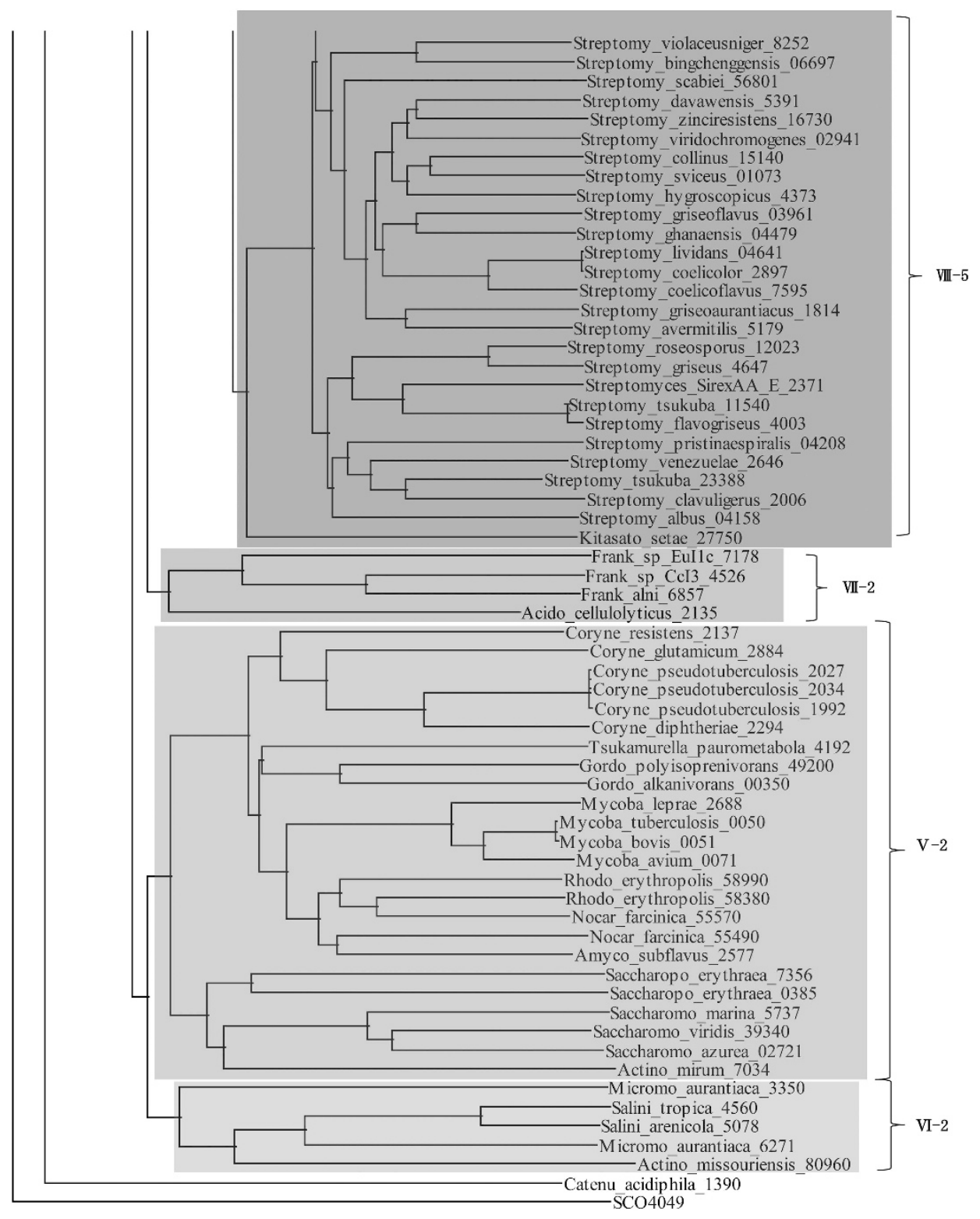

Figure 2 Continued.

(WP_009397921, 4.3e-38). The PBP of S. clavuligerus, SCLAV_4179, is reported to have a low affinity to $\beta$-lactam antibiotics. ${ }^{23}$ Therefore, the low-affinity PBP gene of S. clavuligerus (SCLAV_4179) is supposed to overspread to most Actinobacteria, especially to Streptomyces species, to have a major role in $\beta$-lactam resistance and to reflect on the fact that most Streptomyces species, in particular, are highly resistance to benzylpenicillin, although they are Gram-positive bacteria. ${ }^{25}$ In addition, two low-affinity type PBPs, SCLAV_1774 and SCLAV_4179, and SCAT_1730 and SCAT_5676, greedily present in S. clavuligerus and $S$. cattleya, $\beta$-lactam-antibiotic producers, reinforces their selfresistance to their own $\beta$-lactams.

Another PBP proposed to be involved in the self-resistance in S. clavuligerus is SCLAV_4198.26,27 SCLAV_4198 belongs to subcluster VIII-7 together with SCLAV_2276. Most Streptomyces species occupy two or three PBPs in this subcluster. These PBPs are supposed to strengthen further the self-resistance against $\beta$-lactam antibiotics in these Streptomyces species. Furthermore, most of these PBPs are adjacent to each other, as described above. S. clavuligerus is again an exception.

Goffin and Ghuysen ${ }^{18,26}$ showed that class B PBPs from Grampositive bacteria were classified into three distinct subclasses, B1 (whose prototype is Enterococcus faecium PBP5, X84859), B4 (whose prototype is $S$. pneumoniae $\mathrm{PBP} 2 \mathrm{x}, \mathrm{P} 14677$ ) and $\mathrm{B} 5$ (whose prototype is $S$. pneumoniae PBP2b, P10524). Phylogenetic and similarity analyses indicate that all the PBPs from Streptomyces analyzed in this paper form disparate clusters from these subclass members, and SCLAV_4179 is only distantly related to class B1/B2 PBPs, lowaffinity class PBPs, rather than class B4 or B5 PBPs, whether they are analyzed in whole sequence or penicillin-binding core sequences ${ }^{26}$ (see Supplementary Figure 1).

Protein kinases are classified into two families based on their biochemical similarities and enzymatic specifications as following: the histidine kinase superfamily belonging to the two component systems $^{28}$ and the serine/threonine and tyrosine protein kinase 
superfamily. ${ }^{29,30}$ Recently, these serine/threonine and tyrosine protein kinases were shown to be involved in the regulation of cell morphogenesis of Streptococcus pneumonia, ${ }^{31}$ Corynebacterium glutamicum $^{32}$ and Mycobacterium tuberculosis, ${ }^{33}$ germination of Bacillus subtilis spores ${ }^{34}$ and polar growth and hyphal branching in S. coelicolor. ${ }^{35}$ Although investigating the S. coelicolor homolog of $\mathrm{PknB}$, a serine/threonine protein kinase of $M$. tuberculosis, Yeats et al. ${ }^{36}$ identified a novel domain called PASTA domain that is found in the C-termini of eukaryotic-like serine/threonine kinases and PBPs. This domain binds $\beta$-lactam antibiotics and their peptidoglycan analogs. It is intriguing in this connection that serine/threonine protein kinases are present next to PBPs in Streptomyces species. Furthermore, these protein kinases carry four PASTA domains in tandem in these molecules. Such protein kinases are K530_23511, SSHG_02907, SAV_4338, SBI_05406, SCAT_3089, SCATT_30800, SCLAV_2946, SMCF_8885, SCO3848, B446_19315, BN159_4479, SFLA_3201, SSFG_03588, SGM_3503, SSRG_03159, SGR_3725, SHJG_5218, SSPG_03807, SSDG_03054, SRIM_00070, SrosN1_ 010100017505, SCAB_45561, F750_3547, SACTE_3284, SSEG_02705, STSU_17414, SVEN_3632, STRV1_0274, SSQG_03956 and SZN_17937. However, although these PBPs adjacent to the protein kinases belong to the same subcluster VIII-3, they have no PASTA domain in their molecules in contrast to PBPs in other bacteria. The PBPs in Actinobacteria such as M. tuberculosis class A PBP (accession number is YP_178005, the same hereafter), Rhodococcus sp. DK17 (WP_016884523) and Nocardia sp. BMG111209 (WP_019931711) possess one PASTA domain each in their C-terminal region. Intriguingly, the amino-acid sequences of the protein kinases in this group are almost the same with each other, especially in $\mathrm{N}$-terminal regions containing the protein kinase domains. Protein kinases located adjacent to PBPs are also seen in other Actinobacteria such as Cfla_0025 and Cfla_0026, AMIR_0021 and AMIR_0022, TCUR_0063 and TCUR_0064, Snas_6471 and Snas_6472, and Afer_0087 and Afer_0088. Two protein kinases arrange in tandem, and then comes PBP. Although the function of these protein kinases and the relationship to PBPs are not known yet, they might involve in peptidoglycan biosynthesis in concert with PBPs.

\section{CLASS A PBPS}

A phylogenetic tree constructed on the basis of their amino-acid sequences of 292 class A PBPs from Actinobacteria is classified into 10 clusters and is shown in Figure 2. Like the class B PBPs, the PBPs from taxonomically related species form the same clusters. Accordingly, cluster I consists of PBPs of subclass Rubrobacteridae (Rubrobacter and Conexibacter) and Coriobacteriae (Atopobium, Cryptobacterium and Slackia). All the PBPs of Trophyryma, Clavibacter and Leifsonia compose cluster II. The similarity of the amino-acid sequences between TW_0722 and TWT_0705 is 100\% except the C-terminal amino acid, where glutamic acid is replaced by aspartic acid. Aminoacid sequence similarity values (E-values) are $1.3 \mathrm{e}-68$ between TW_0722 and CMM_0915, 1.2e-86 between TW_0722 and LXX_03600, and 2.5e-70 between TW_0722 and LXX_02090. Among Micrococcineae PBPs, PBPs of three genera (Trophyryma, Clavibacter and Leifsonia) behave as a group like the class B PBPs. Cluster III is made up of PBPs of order Bifidobacteriales (Bi ${ }^{\circledR}$ dobacterium and Gardnerella). These PBPs are divided into two subclusters, III-1 and III-2. PBPs of suborder Actinomycineae (Actinomyces and Mobiluncus) and Micrococcineae (Isoptericola, Beutenbergia, Brevibacterium, Micrococcus, Rothia, Kocuria, Janibacter, Renibacterium and Arthrobacter), together with two PBPs of genus Kineococcus (KRAD_0429 and KRAD_4341), form subclusters IV-1, IV-2 and 
Streptomy_rimosus_ 22689 Kitasato_setae_38960 Streptomy_scabiei_64431 Streptony_zinciresistens_18682 Streptony_viridochromogenes_02 Streptomy_ghanaensis_02387 Streptony_griseoaurantiacus_055 Streptomy_avermitilis_7219 Streptomy_hygroscopicus_3853 Kitasato_setae_59840 Streptony_rimosus_08328 Streptony_violaceusniger_2314 Streptomy_bingchenggensis_03076

Streptomy_rimosus_2268 Kitasato_setae_38960 Streptomy_scabiei_64431 Streptomy_zinciresistens_18682 Streptomy_viridochromogenes_0232 Streptony_ghanaensis_02387 Streptony_griseoaurantiacus_0550 Streptony_avermitilis_7219 Streptony_hygroscopicus_ 3853 Kitasato_setae_59840 Streptony_rimosus_083 Streptony_rimosus_08328 Streptony_violaceusniger_2314
Streptomy_bingchenggensis_03076

Streptony_rimosus_2268 Ki tasato_setae_38960 Streptony_scabiei_6443 Streptony_zinciresistens_18682 Streptony_viridochromogenes_0232 Streptony_ghanaensis_02387 Streptomy_griseoaurantiacus_0550 Streptony_avermitilis_7219 Streptomy_hygroscopicus_3853 Kitasato_setae_59840 Streptony_rimosus_08328 Streptony_violaceusniger_2314 Streptony_bingchenggensi s_03076

Streptomy_rimosus_22689 Kitasato_setae_38960 Streptomy_scabiei_64431 Streptomy_zinciresistens_18682 Streptomy_viridochromogenes_0232 Streptomy_ghanaensis_02387 Streptomy_griseoaurantiacus_0550 Streptomy_avermitili is_7219 Strept tomy_hygroscopicus_3853 Kitasato_setae_59840 Streptony_rimosus_08328 Streptomy_rimosus_08328 Streptomy_bingchenggensis_03076

Streptomy_rimosus_22689
Ki tasato_setae_38960
Streptony_scabiei_64431
Streptomy_zinciresistens_18682
Streptony_viridochromogenes_0232
Streptony_ghanaensis_02387
Streptomy_griseoaurantiacus_0550
Streptony_avermiti1iis_7219
Streptomy_hygroscopicus_3853
Kitasato_setae_59840
Streptomy_rimosus_08328
Streptomy_violaceusniger_2314
Streptony_bingchenggensis_03076

Streptony_rimosus_22689 Kitasato_setae_38960 Streptomy_scabiei_64431 Streptomy_scabiei_64431 Streptomy_viridochromogenes_0232 Streptomy_viridochromogenes_ Streptomy_ghanaensis_02387
Streptomy_griseouurantiacus_0550 Streptomy_avermitilis_7219 Streptomy_hygroscopicus_3853 Ki tasato_setae_59840 Streptomy_rimosus_08328 Streptomy_violaceusniger_2314 Streptomy_bingchenggensis_03076

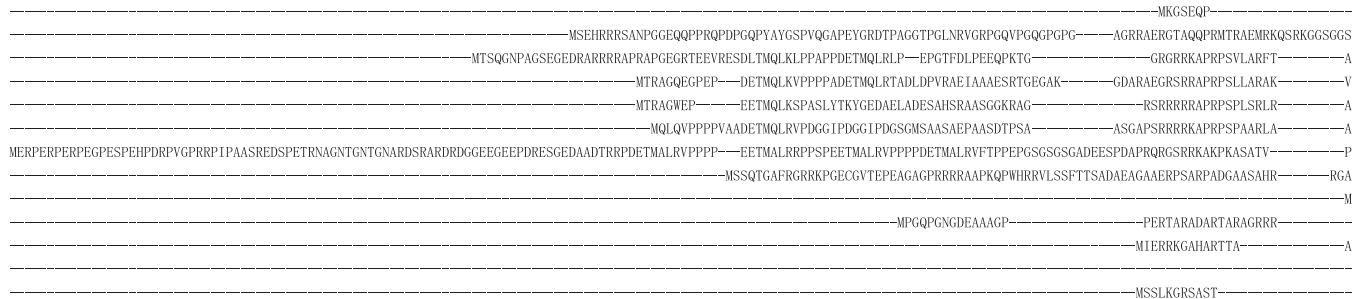

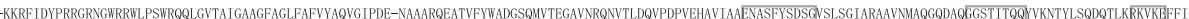
CCCM

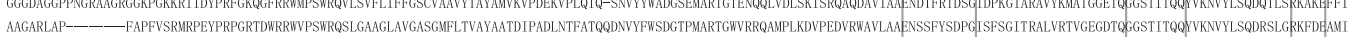

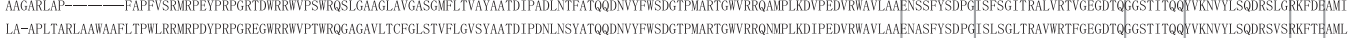

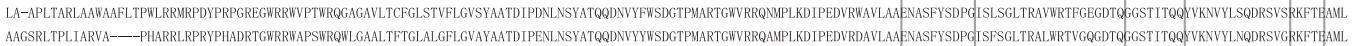

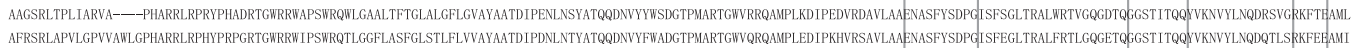

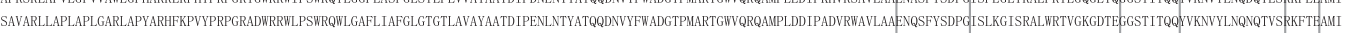
RRKAAEPTAIARVTVRLTPYARRLKPEYPRPGLDGWRRWMPVRQWLGICLVSLGLSGTFLAVAYAATDIPDNLNTYATQQDNVYFWADGTPMARTGWVRRQAMPLKDIPEDVRWAVLAAEVASFYSDPGISVSGIGRALWRTVGEGDTQGGSTT TQQYVKNVYLTQNRSVTRKFTBAMI GAVVRLLGPLAPYARRIAPYARRLKPYYPRPGRTGWRRWMPNRQWLGGATLTSFGLLSAFLVTAYALTDIPENLNSYATQQDNVYFWSDGTPMARTGWVQRQAMPLKDIPPDVRWAVLAAEVESFYSDPGISARGITRAVFRTLGQGDTEGGSTITQQYVKNVYLTQNQTISRKRTEAMI

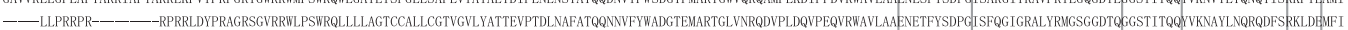
APDGRWPALLLRWWRAGYLRIRRLRPAYPRPGRTGLRRWIPSWRQSLGLGGSFVG LLTGLLAIYYARTEIPADLNAFATQQDNYYYWADGTEMARTGDINRQDMPLDSVPEKVRWMLLMENETFYSDSGISPSG I IRAATRMVTGGDTOGGSTITQQVVKNAYLNQDQTFTRKLTBAFI

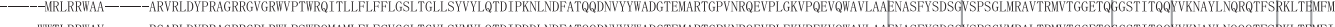

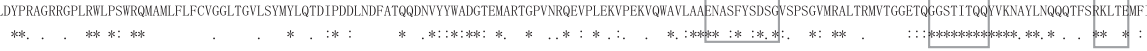

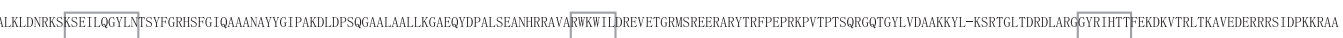

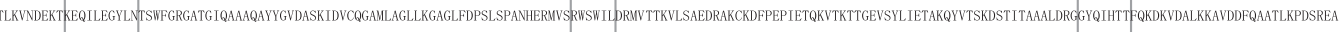
ALKLDVYQMSKDEILEGYLNTSWFGRGTYG IQRASQAYYGKDVSELNVSEAAALASLLKGGGLYDPSLSRANHARTVVRWEW ILDRUVAIKKLSKAERAKYTKFPEPLDPSNQYDTGKQSDYLVELASQYA-KKAANITAQEFDRGGYQIYTTFDKDRQTELTDAVTKAR-REVTEKNPKK

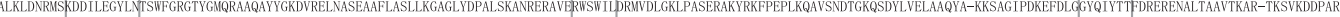
ALKLDVGMSKDDILEGYLNTSWFGRGTYGIQRAAQAYYGKDVGELDAGEAAFLASLLKGASLYDPALSKANHARAVERWSWILDRAVDNGMLSKSERAKYKEFPEPLKQTPGYDTGKQSDYLVELMAQYA-KKAHHISAKEFDLGGFQIYTTFDRKQETALTDAVAEAR-KKARKDDAMK

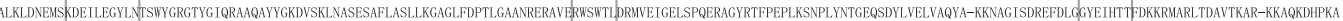

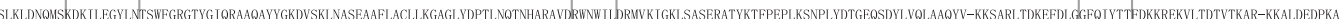
WW ALKLDVRMSKDQILEGYLNTSWFGRGSYG IORAAQAYYGKDVDKLVASEAAMLAGLLKGAGLFDPTLSKANHARAVEPVSWID DRUVKIGKLSPQERAKYTTFPEPLKSNPLYNTGAQSDYLVELASQYA-KKGGHLTDKOFDLGGYQIYTTFDRRREKALTDAVTKAR-KQARQDNPAA

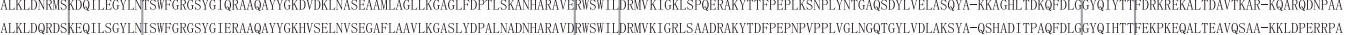

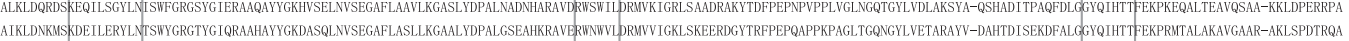

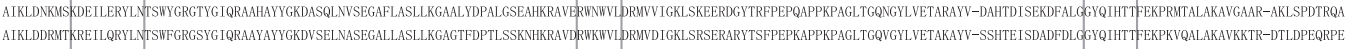

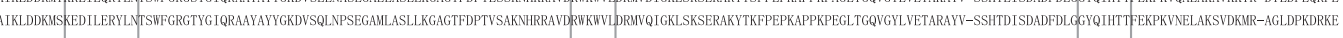

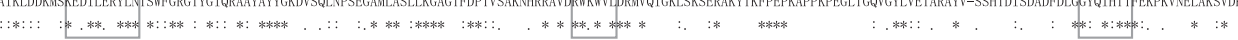

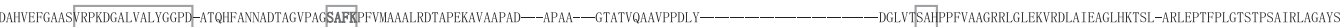
DKFVQVGAASVLPGDGAIVAIYGGPGFENNHYTNADARGGVYGSTFKPIVLAAAMODGVLTKNGEDGKPAQINPDSRYLADDWAQIYRADGSKAMENGQPYHORNSDPGKKGYVTLRTAMQNSYNVPFVQLGQDVGGDKVRAMAQTLGLLDDTLLAGASTLTLPLGTSTPSA IRAASYYS DKFVQVGAASLLPGDGAIVAIYGGPGFENNHYTNNADAKGVPVGSTFKPIVLAAAMQDGVLTKNGEDGKPAQINPDSRYLADDUAQIYRADGSKAMENGQPYHQRNSDPGKKGYVTLRTAMQNGYYVPFVQLGGDVGGDKVVAMAQTLGLLDDDTLAGASTLTLPLGTSTPSAIRUASVYS

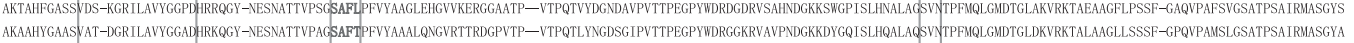

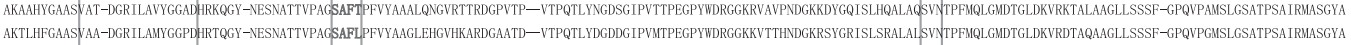
ARTAHYGAASIAA-DGRILAVYGGPDHRRgGY-VESNATTVPAGSAFMPFVYAGGLEHGVRKTRDGERTP-VVTGETVYNGDDEVPVTTPEGPYWDRGGRKVSARVDGGKSYGQISLRRALAISVITPFMQLGMDTGLDKVRATAVAAGLLPSSI-GARVPALSTGSSTPSAIRMAGGYA KRTAHYGASIIAS-DGRVIAVYGGPDHRTQGY-NESNATTVPAGTAFLPFVYAAGLEHGVTRKRDAPRTP-VTPESVYDGDDGVPVTTPEGPYWDRSGKKVAAHNDGKKDWGRISLGKALAHSVITPFMQLGMDTGLDNVRDTAVAAGLLSSSM-GAQVPDLSLGSSRPSA IRMASAYA AETAHYGGSSVAA-DGRILAVYGGPDHRRQGF-NESNASTVPAGSAFLPFVYAAALEHGVIKDRGSTTTP-VTPDSVYNGDDGVPVTTPEGPYWDRSGKKVAAHNDGGTSWGQITLRKALARSVYTPFMQLGMDGGLATVRETAEATGMLSSSM-GPQVPTLSLGTSTPSAIRMASSYA AKDGHYGMSYMA-DGR LLAVYGGPDHRTQGY-NESNATTVPAGTAFTPFVYAAGLEHGVHKTRGGPATP-VVTPDSVYDGDDGVPVTTPEGPYWDSGGKKVTAHNDGGRSYGDISLREMMARSVITPFMQLGMDTGLDKVRQTAVASGLLSSSI-GPRVPALSMGNSTPSAIRMAGGYA

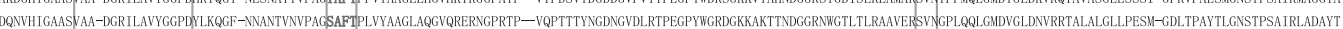

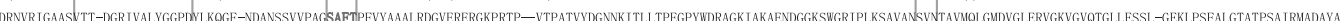
D

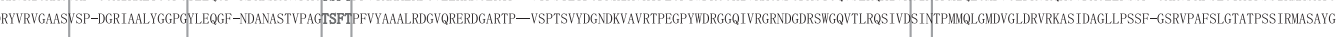

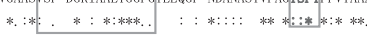

LFGNDGSRTEPYSVTRVEHDGTPLPGLTPPAPRRVLTPGAAHGINWALR-AYAGKTLPPETVAKLPRGIWAG VFAAHGQQTDPYSVTAVQFHIGKDLPNFARPTPKTALDPAVADNITDVLQQNVAKN-GTGNKTAALGFPV-AGTFAMQGHTEPYSVRKMTHNGYRVP-LKTPGPERAVGADVMAVTSALTDAFRT--AHPDSPGASYYV-MATFAAEGNHVEPYSVSRITRNGSKVA-LERPDRRRAMSPQVAEQVQSALRDSLRL--ADPAAADSAP-QA-AG- TFAAGGTHVEPYSVARITRNGSEVR-LAKPASRRAVRASVAASVQSALTDAFRT-ARPGADPDT--EV-AG- TFAADGLHTEPYSVRRITHNGHKVA-LAEPRTRRAMGADTAREVQAALADAFRE-AHPEDAPATA-EV-AGTFDAGGRHTEPYSVRRVTHNGSTVP-MIDPPAVRRAMGADVAGQVTEALTDSFHL-AHPTATAASA-KV-SG TFAGGTHTAPYSVRRVTRNGSKVD-LDTPRRRRAVGADVARQVTDALTDSFRT--QHPTAMPASA-QV-AG-

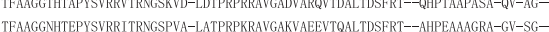

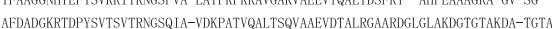

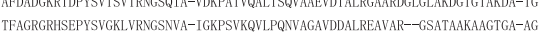
TFAGRGRHSEPYSVGKLVRNGSNVA-IGKPSVKQVLPQWVAGAVDDALREAVAR--GSATAAKAAGTGA-AG TFAaGGRHTPPYSVTGLTHQGERVP-LEKQPATRAFSPEVAaVDDALHGAVQG-GTAQSAAAVGSDV-AG-

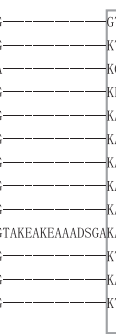
GTGPDRURSAWF IGNDGTRGGGLTTAVTVFRAEP-GAPRLLPMEGVGGD-*. : **:**:*. *

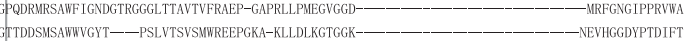

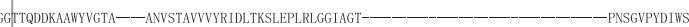

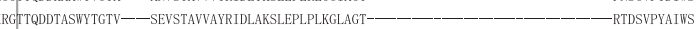

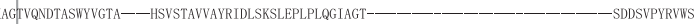

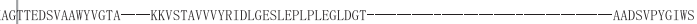

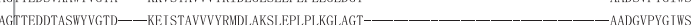

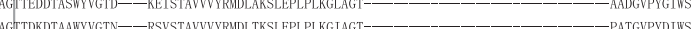
PATGPYIWS A A SASPYALWS

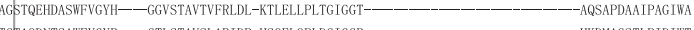

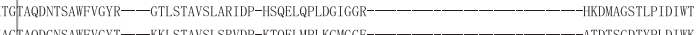

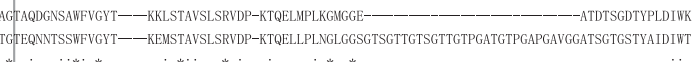
$*::: *: * \quad: *:: \quad *: \quad: \quad: * *$ AY----ATG--RYM---KIIGPGNPRNFTPPTPWGQEVDSSGAPVSASPSASATESAPAVPTEQPSVPVEQPSPTESASSTGKPTGKPSGSPTASPSTCLPVICNPGTTGGTGTTGGTGTTTGTNNGGTNGTPTGTPTGTGTTTGRT RAM---NIG--_-_RAM---NIG---

GALGLDRPVDRSS-

GA----RGLG

RAM---SPLG

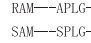

SAM---SPLG-

AA----TGLG-

RYM---TAR-

SYM-TDRAAE-

YYY---KAYTPPS------

Figure 3 Amino-acid alignment of 13 PBPs in subcluster VIII-3 of the phylogenetic tree (Figure 2). The amino-acid sequences are aligned by using MUSCLE. ${ }^{41}$ The conserved motifs ${ }^{18}$ are boxed, and the essential SXXK sequences are marked with red, bold letters. A full color version of this figure is available at The Journal of Antibiotics journal online. 
Table 4 Low-affinity and some closely related PBPs in Streptomyces

\begin{tabular}{lc}
\hline Penicillin-binding protein & E-value (similarity value) \\
\hline Subcluster VIII-6 & \\
Streptomy_clavuligerus_4179 & $7.2 \mathrm{e}-218$ (100\%) \\
Streptomy_clavuligerus_1774 & $1.4 \mathrm{e}-145$ \\
Streptomy_albulus_21226 & $3.8 \mathrm{e}-156$ \\
Streptomy_bingchenggensis_07119 & $4.1 \mathrm{e}-186$ \\
Streptomy_cattleya_5676 & $8.4 \mathrm{e}-186$ \\
Streptomy_coelicolor_2608 & $1.1 \mathrm{e}-160$ \\
Streptomy_davawensis_5684 & $1.1 \mathrm{e}-167$ \\
Streptomy_griseoflavus_04177 & $3.1 \mathrm{e}-167$ \\
Streptomy_griseus_4934 & $4.5 \mathrm{e}-165$ \\
Streptomy_hygroscopicus_4100 & $2.9 \mathrm{e}-163$ \\
Streptomy_scabiei_60051 & $1.7 \mathrm{e}-174$ \\
Streptomy_venezuelae_2386 & $3.7 \mathrm{e}-172$ \\
& \\
Subcluster VIII-7 & \\
Streptomy_clavuligerus_4198 & \\
Streptomy_clavuligerus_2276 & $5.5 \mathrm{e}-144(100 \%)$ \\
Streptomy_albulus_01507 & $6.3 \mathrm{e}-34$ \\
Streptomy_bingchenggensis_02283 & $2.8 \mathrm{e}-28$ \\
Streptomy_cattleya_0768 & $6.3 \mathrm{e}-30$ \\
Streptomy_coelicolor_3156 & $6.7 \mathrm{e}-26$ \\
Streptomy_davawensis_5121 & $2.5 \mathrm{e}-30$ \\
Streptomy_griseoflavus_03076 & $1.8 \mathrm{e}-30$ \\
Streptomy_griseus_4340 & $1.9 \mathrm{e}-41$ \\
Streptomy_hygroscopicus_4628 & $1.1 \mathrm{e}-27$ \\
Streptomy_scabiei_53621 & $8.5 \mathrm{e}-32$ \\
Streptomy_venezuelae_2985 & $2.5 \mathrm{e}-25$ \\
\hline & $1.3 \mathrm{e}-24$ \\
\hline
\end{tabular}

IV-3. The class A PBPs of Kineococcus radiotolerans behave with those of suborder Micrococcineae, such as Brevibacterium, but other PBPs of suborder Micrococcineae, such as Isoptericola and Beutenbergia, comport themselves with those of suborder Actinomycineae, such as Actinomyces and Mobiluncus (subclusters IV-1 and IV-3). PBPs of suborder Corynebacterineae (Corynebacterium, Tsukamurella, Gordonia, Mycobacterium, Rhodococcus, Nocardia and Amycolicicoccus) and Pseudonocardineae (Saccharopolyspora, Saccharomonospora and Actinosynnema) form subclusters V-1 and V-2 but different branches, although the amino acid similarity is not so different between PBPs of these branches. PBPs of genus Nakamurella move with those of suborder Pseudonocardineae like the class B PBPs, although genus Nakamurella is classified as suborder Frankineae. Subclusters VI-1 and VI-2 consist of PBPs of suborder Micromonosporineae (Salinispora, Micromonospora and Actinoplanes). PBPs of genus Stackebrandtia form outgroups in the phylogenetic tree as suggested by the taxonomic position. PBPs of genus Frankia form distinct subclusters VII-1 and VII-2, and those of genus Propionibacterium construct other discrete subclusters IX-1 and IX-2.

Like the class B PBPs, class A PBPs of Streptomyces species form large five subclusters, VIII-1, VIII-2, VIII-3, VIII-4 and VIII-5. Similarity analyses of class A PBPs from Streptomyces indicate that those inherent in the same subcluster have very low E-values irrespective of different species, especially PBPs in subcluster VIII-1 (Table 3). In addition, E-values between PBPs belonging to different subclusters but from the same species are not so different from those from different species. The amino-acid sequence similarity among class A PBPs are generally higher than those among class B PBPs. Another interesting fact clarified by amino-acid alignment analysis is that among 13 PBPs in subcluster VIII-3, 4 PBPs (SGM_0550,
SHJG_3853, STRVI_2314 and SBI_03076) do not have essential serine residues in the motif SXXK. In addition, except two PBPs (SRIM_22689 and KSE_38960), they do not possess essential lysine residues, although other features ${ }^{18,26}$ requisite for PBPs are conserved (Figure 3), suggesting that it is doubtful whether these PBPs function as transpeptidases or the transpeptidase activity is very low even though they retain penicillin-binding properties.

Class A PBPs from Gram-positive bacteria are classified into five subclasses, ${ }^{26}$ A1 (whose prototype is Escherichia coli PBP1A), A2 (whose prototype is E. coli $\mathrm{PBP} 1 \mathrm{~B}$ ), A3 (whose prototype is Streptococcus pneumoniae 1A), A4 (whose prototype is $S$. pneumoniae $2 \mathrm{~A}$ ) and A5 (whose prototype is $S$. pneumoniae 1B). Phylogenetic and similarity analyses indicate that all the class A PBPs from Streptomyces analyzed in this paper form a completely different cluster in a phylogenetic tree from these five clusters, where E-values are in the range of $3.4 \mathrm{e}-16$ to $4.9 \mathrm{e}-31$, indicating very low similarities (see Supplementary Figure 2). These results, together with the results in class B PBPs where E-values range from 9.3 to $7.7 \mathrm{e}-39$, suggests strongly that the gene transfer and/or gene conversion occurred very rarely between PBPs in Streptomyces and those in Gram-positive and Gram-negative bacteria.

\section{PBPS WITH LOW AFFINITY TO PENICILLINS}

Ogawara and Horikawa ${ }^{37}$ reported over 30 years ago that $\beta$-lactamproducing Streptomyces species possessed PBPs of very low affinity to benzylpenicillin. Later, two PBPs, that is, SCLAV_4179 and SCLAV_4198 were reported to have low affinity to penicillins..$^{23,27}$ A mutant disrupted in SCLAV_4198 gene exhibited a significant decrease in its resistance to benzylpenicillin and cephalosporins. ${ }^{27}$ Moreover, a probe containing SCLAV_4198 hybridized to genomic DNAs from $\beta$-lactam producers, S. jumonjinensis NRRL 5741, S. griseus NRRL 3851 and S. lipmanii NRRL 3584, suggesting that SCLAV_4198-like sequences and SCLAV_4198-mediated resistance mechanisms are likely to be present in these $\beta$-lactam-producing species. Table 4 lists low-affinity PBPs and some of the closely related PBPs in Streptomyces. The PBPs belonging to subclusters VIII-6 and VIII-7 in Figure 1 are assumed to have low affinity to penicillins.

\section{CONCLUSION}

The work on self-resistance to $\beta$-lactam antibiotics in Actinobacteria in my research career started by the findings that most of the Streptomyces species constitutively produced $\beta$-lactamase independent of their resistance to $\beta$-lactam antibiotics and $\beta$-lactam production, ${ }^{25,38}$ and the detection of PBPs in Streptomyces species by autoradiography took over 6 months instead of a few days in E. coli ${ }^{16}$ and B. subtilis. ${ }^{37}$ When I visited Dr Hamao Umezawa, my boss at that time, for the proofreading of the paper, he immediately said that 'The avoidance of the contamination of Streptomyces species was the most important and absolute necessity in the fermentation of Penicillium for the production of benzylpenicillin. It caused the complete destruction of benzylpenicillin because of their production of $\beta$-lactamases.' $\mathrm{He}$ knew by experience that most Streptomyces species produced $\beta$-lactamases. On the basis of these two findings, I proposed about 35 years ago in Antimicrobial Agents and Chemotherapy ${ }^{37}$ and Microbiological Reviews ${ }^{39}$ that low-affinity PBPs were the main cause of self-resistance to $\beta$-lactam antibiotics in Streptomyces. Since then, supporting evidence is gradually accumulating. This review offers some substantiating evidence from the points of PBPs for selfresistance and resistance in general to $\beta$-lactam antibiotics in Streptomyces even though they are Gram-positive bacteria. 
1 Fleming, A. On the antibacterial action of cultures of a penicillium, with special reference to their use in the isolation of B. influenza. Br. J. Exp. Pathol. 10, 226-236 (1929).

2 Bush, K. \& Jacoby, G. A. Updated functional classification of $\beta$-lactamases. Antimicrob. Agents Chemother. 54, 969-976 (2010).

3 Spratt, B. G. The 2011 Garrod Lecture: from penicillin-binding proteins to molecular epidemiology. J. Antimicrob. Chemother. 67, 1578-1588 (2012).

4 Gao, B. \& Gupta, R. S. Phylogenetic framework and molecular signatures for the main clades of the phylum Actinobacteria. Microbiol. Mol. Biol. Rev. 76, 66-112 (2012).

5 Ventura, M. et al. Genomics of Actinobacteria: tracing the evolutionary history of an ancient phylum. Microbiol. Mol. Biol. Rev. 71, 495-548 (2007).

6 Chater, K. F., Biró, S., Lee, K. J., Palmer, T. \& Schrempf, H. The complex extracellular biology of Streptomyces. FEMS Microbiol. Rev. 34, 171-198 (2010).

7 Liras P., Martin J. F. Gene clusters for $\beta$-lactam antibiotics and control of thei expression: why have clusters evolved, and from where did they originate?. Int Microbiol. 2006; 9: 9-19.

8 Ochi, K., Hosaka, T. New strategies for drug discovery: activation of silent or weakly expressed microbial gene clusters. Appl. Microbiol. Biotechnol. 97, 87-98 (2013).

9 Núñez, L. E., Méndez, C., Braña, A. F., Blanco, G. \& Salas, J. A. The biosynthetic gene cluster for the $\beta$-lactam carbapenem thienamycin in Streptomyces cattleya. Chem. Biol. 10, 301-311 (2003).

10 Cundliffe, E. How antibiotic-producing organisms avoid suicide. Annu. Rev. Microbiol. 43, 207-233 (1989)

11 Cundliffe E. \& Demain A. L. Avoidance of suicide in antibiotic-producing microbes. J. Ind. Microbiol. Biotechnol. 37: 643-672 (2010).

12 Davies, J. \& Davies, D. Origins and evolution of antibiotic resistance. Microbiol. Mol. Biol. Rev. 74, 417-433 (2010).

13 Thaker, M. N. et al. Identifying producers of antibacterial compounds by screening for antibiotic resistance. Nat. Biotechnol. 31, 922-927 (2013).

14 Macheboeuf, P., Contreras-Martel, C., Job, V., Dideberg, O. \& Dessen, A. Penicillin binding proteins: key players in bacterial cell cycle and drug resistance processes. FEMS Microbiol. Rev. 30, 673-691 (2006).

15 Typas, A., Banzhaf, M., Gross, C. A. \& Vollmer, W. From the regulation of peptidoglycan synthesis to bacterial growth and morphology. Nat. Rev. Microbiol. 10 , 123-136 (2012).

16 Spratt, B. G. Distinct penicillin binding proteins involved in the division, elongation, and shape of Escherichia coli K12. Proc. Natl Acad. Sci. USA 72, 2999-3003 (1975).

17 Suginaka, H., Blumberg, P. M. \& Strominger, J. L. Multiple penicillin-binding components in Bacillus subtilis, Bacillus cereus, Staphylococcus aureus, and Escherichia coli. J. Biol. Chem. 247, 5279-5288 (1972).

18 Goffin, C. \& Ghuysen, J.-M. Biochemistry and comparative genomics of SxxK superfamily acyltransferases offer a clue to the mycobacterial paradox: presence of penicillinsusceptible target proteins versus lack of efficiency of penicillin as therapeutic agent. Microb. Mol. Biol. Rev 66, 702-738 (2002).

19 Pratt, R. F. Substrate specificity of bacterial DD-peptidases (penicillin-binding proteins). Cell Mol. Life Sci. 65, 2138-2155 (2008).

20 Sauvage, E., Kerff, F., Terrak, M., Ayala, J. A. \& Charlier, P. The penicillin-binding proteins: structure and role in peptidoglycan biosynthesis. FEMS Microbiol. Rev. 32 234-258 (2008).

21 Ogawara, H. Self-resistance to $\beta$-lactam antibiotics in Streptomyces (in Japanese). Bull. Meiji Pharmaceut. Univ 43, 1-20 (2014).
22 Zhi, X. Y, Li, W. J. \& Stackebrandt, E. An update of the structure and 16S rRNA gene sequence-based definition of higher ranks of the class Actinobacteria, with the proposal of two new suborders and four new families and emended descriptions of the existing higher taxa. Int. J. Syst. Evol. Microbiol. 59, 589-608 (2009).

23 Ishida, K. et al. Characterization of $p b p A$ and $p b p 2$ encoding penicillin-binding proteins located on the downstream of clavulanic acid gene cluster in Streptomyces clavuligerus. Biotechnol. Lett. 28, 409-417 (2006).

24 National Institutes of Health Protein clusters: PCLA_902386. Available at http://www. ncbi.nlm.nih.gov/proteinclusters/?term = PCLA_902386. Accessed on 24 September 2014.

25 Ogawara, H. Production and properties of beta-lactamase in Streptomyces. Antimicrob. Agents Chemother. 8, 402-408 (1975).

26 Goffin, C. \& Ghuysen, J.-M. Multimodular penicillin-binding proteins: an enigmatic family of orthologs and paralogs. Microb. Mol. Biol. Rev. 62, 1079-1093 (1998).

27 Paradkar, A. S, Aidoo, K. A, Wong, A. \& Jensen, S. E. Molecular analysis of a $\beta$-lactam resistance gene encoded within the cephamycin gene cluster of Streptomyces clavuligerus. J. Bacteriol. 178, 6266-6274 (1996).

28 Hoch, J. A. \& Varughese, K. I. Keeping signals straight in phosphorelay signal transduction. J. Bacteriol. 183, 4941-4949 (2001).

29 Molle, V. \& Kremer, L. Division and cell envelope regulation by Ser/Thr phosphorylation: Mycobacterium shows the way. Mol. Microbiol. 75, 1064-1077 (2010).

30 Urabe, H. \& Ogawara, H. Cloning, sequencing and expression of serine/threonine kinase-encoding genes from Streptomyces coelicolor A3(2). Gene 153, 99-104 (1995).

31 Morlot, C. et al. Interaction of penicillin-binding protein $2 x$ and Ser/Thr protein kinase StkP, two key players in Streptococcus pneumoniae R6 morphogenesis. Mol. Microbiol. 90, 88-102 (2013).

32 Fiuza, M. et al. Phosphorylation of a novel cytoskeletal protein (RsmP) regulates rodshaped morphology in Corynebacterium glutamicum. J. Biol. Chem. 285, 29387-29397 (2010).

33 Munshi, T. et al. Characterisation of ATP-dependent Mur ligases involved in the biogenesis of cell wall peptidoglycan in Mycobacterium tuberculosis. PLOS ONE 8, e60143 (2013).

34 Shah, I. M., Laaberki, M. H., Popham, D. L. \& Dworkin, J. A eukaryotic-like Ser/Thr kinase signals bacteria to exit dormancy in response to peptidoglycan fragments. Cell 135, 486-496 (2008).

35 Hempel, A. M. et al. The Ser/Thr protein kinase AfsK regulates polar growth and hyphal branching in the filamentous bacteria Streptomyces. Proc. Natl Acad. Sci. USA 109, E2371-E2379 (2012).

36 Yeats, C., Finn, R. D. \& Bateman, A. The PASTA domain: a $\beta$-lactam-binding domain. Trends Biochem. Sci. 27, 438-440 (2002).

37 Ogawara, H. \& Horikawa, S. Penicillin-binding proteins of Streptomyces cacaoi, Streptomyces olivaceus, and Streptomyces clavuligerus. Antimicrob. Agents Chemother. 17, 1-7 (1980).

38 Ogawara H., Horikawa S., Shimada-Miyoshi S. \& Yasuzawa K. Production and properties of $\beta$-1actamases in Streptomyces.Comparison of the strain isolated newly and thirty years ago. Antimicrob. Agents Chemother. 1978; 13: 865-870.

39 Ogawara $\mathrm{H}$. Antibiotic resistance in the pathogenic and the producing bacteria, with special reference to $\beta$-lactam antibiotics. Microbiol. Rev. 1981; 45: 591-619.

40 Larkin, M. A. et al. Clustal W and Clustal X version 2.0. Bioinformatics 23, 2947-2948 (2007).

41 Edgar, R. C. MUSCLE: multiple sequence alignment with high accuracy and high throughput. Nucleic Acids Res. 32, 1792-1797 (2004).

Supplementary Information accompanies the paper on The Journal of Antibiotics website (http://www.nature.com/ja) 